XIV.

\title{
41. Wanderversammlung der Siidwestdeutschen Neurologen und Irrenärzte am 3. und 4. Juni 1916 in Baden-Baden.
}

Anwesend sind die Herren:

Allendorf-Karlsruhe, Assmann-Leipzig, zurzeit Karlsrube, L. Auerbach-Frankfurt a. M., Barbo-Pforzheim, BayerthalWorms, Bäumler-Freiburg, Beetz-Stuttgart, Becker-BadenBaden, Bergenthal-Düsseldorf, Berliner-Giessen, Bethe-Frankfurt a. M., Beyer-Roderbirken b. Leichlingen, BöckelmannStephansfeld, Böhs-Wiesloch, Buttersack-Heilbronn, de la CampFreiburg, Damköhler-Günzburg, Deetjen-Wilhelmshöhe, Dinkler-Aachen, Dillenburger-Strassburg, G. L. Dreyfus-Frankfurt a.M., Ebers-Baden-Baden, Edinger-Frankfurta. M., W. ErbHeidelberg, Eschbanm-Cöln, Fahrenkamp-Heidelberg, Feldbausch-Emmendingen, Finkelnburg-Bonn, M. Fischer-Wiesloch, Gaupp-Tübingen, Gerhardt-Würzburg, Gierlich-Wiesbaden, Giese-Baden-Baden, Groetzer-Baden-Baden, GrossSchussenried, Grü ner-Baden-Baden, Ha ard t-Emmendingen, Hag mann-Coblenz, B. Hahn-Baden-Baden, Hauptmann-Freiburg, Hauser-Mannheim, Haymann-Konstanz, r. Hess-München, Heins heimer-Baden-Baden, Hezel-Wiesbaden, Hoche-Freiburg, Hoestermann-Heidelberg, zurzeit Karlsrube, Hübner-BadenBaden, Hübner-Bonn, Hü gel-Kilingenmünster, Jahnel-Frankfurt a. M., Jolly-Halle, F. Ka ufmann-Mannheim, Ke hr-Hamburg, Kehrer-Freiburg-Bärenstein, Kispert-München, König-Bonn, Le va - Strassburg, H. Lery - Stuttgart, Lot mar - Bern, zurzeit BadenBaden, L. Mann-Mannheim, Meitzen-Wiesbaden, M. Meyer-Köppern i. S., Mörchen-Wiesbaden, Naegeli-Tübingen, NaunynBaden-Baden, Neumann-Karlsruhe, Nissl-Heidelberg, NonneHamburg, Ob kirch er-Baden-Baden, Oster-Konstanz, P fersd orffStrassburg, Pl etzer-Bonn, G. Rath-Baden-Baden, Rieder-Coblenz, Rosenfeld-Strassburg, Saenger-Hamburg, Sohmidt-Freiburg, Schmidt-Mainz, Schoenborn-Heidelberg, Scholz-Strassburg, Schulze-Bonn, Schüle-Illenau, Schwidop-Karlsruhe, zurzeit Chefarzt Baden-Baden, Steiner-Strassburg, Stolzenburg-Göt- 
tingen, Tu ozeck - Marburg, Voss - Düsseldorf, W alle n berg-Danzig, Weichbrodt-Frankfurt a. M., M. Weil-Stuttgart, WeintraudWiesbaden, Westphal-Bonn, Wollenberg-Strassburg, ZacherBaden-Baden.

Folgende Herren haben die 'Versammlung begrüsst, bzw. ihr Fernbleiben entschuldigt:

Buder - Winnental, Duda, Korpsarzt, XV. Res.A.K., ErlenmeyerBlendorf, Eschle-Sinsheim, Friedländer-HoheMark, v. Hecker, Obergeneralarzt, Hoffmann-Heidelberg, Kreuser-Winnental, C. v. Monakow-Zürich, v. Rad-Nürnberg, Römheld-Hornegg, Romberg-München, v. Strüm pell-Leipzig.

\section{Sitznng am 3. Jıni, nachmittags 2 Uhr.}

Der Geschäftsführer Prof. Dinkler-Aachen eröffnet dio Versammiung und begrüsst die Anwesenden. Er gedenkt der im letzten Jahre verstorbenen Prof. Alzbeimer-Breslau, Prof. Schwalbe-Strassburg and Hofrat Weizs äcker-Wildbad, zu deren Ehren sich die Versammlung von ihren Sitzen erhebt.

Zum Vorsitzenden der ersten Sitzung wird Prof. Nonne-Hamburg, für: die zweite Sitzung Geheimrat Hoche-Freiburg gewählt.

Schriftührer: Privatdozenten Hauptmann-Freiburg und SteinerStrassburg.

Es halten Vorträge:

1. Herr Nonne-Hamburg: "Ueber kliniscbe Bilder von Tumoren des Conus terminalis mit negativem anatomischen Befund."

In einem Falle, der 3 Jahre die charakteristischen klinischen Symptome einer Erkrankung des Conus - Cauda-Gebiets bot, und bei dem ätiologisch nichts zu eruieren war, fand sich bei der Sektion makroskopisch und mikroskopisch am Rückenmark und an den Cauda-Wurzeln keine palpable Anomalie. N. bespricht die einschlägige Literatur, dio neueren Erfahrungen über Erkrankung des Conus (Oppenheim, Elsberg, Nonne, Adler, Kurt Mendel, Hans Curschmann, Ornstein), ferner die Fälle von spontaner Rückbildung klinischer Bilder von Kompression des Rückenmarks (Boettiger, Eduard Müller, Henschen, Bruns, Oppenheim, Kredel, Maass, Nonne), rekapituliert die Erfahrungen über Pseudo-Tumor cerebri und erörtert die Frage, ob es angesichts solcber Fälle, wie des jetzt von N. beschriebenen, gerechtfertigt ist, von Pseudo-Tumor spinalis zu sprechen.

(Eigenbericht.)

2. Herr Wollenberg-Strassburg: "Ueber funtrtionelle Extremitätenlähmungen (mit Krankenvorstellung). "

Es wurden vorgestellt zuerst 3 Fälle von schlaffer Lähmung, und zwar einer, in dem die rechte Hand, je einer, in dem der rechte und der linke Arm 
betrofien war. In allen Fällen war die eloktrische Erregbarkeit normal, und bestanden ausgesprochene Störungen der Sensibilität und der Vasomotoren. Immer entsprach die Lähmung der Körperstelle, an welcher die Verwundung stattgefunden hatte. Die Erkrankung war in 2 Fällen ausgesprochen monosym. ptomatisch. In einem Falle, der sich durch beșonders ausgedehnte Sensibilitätsstörung auszeichnete, bestand eine auffällige gemütliche Erregbarkeit, und hier hatte sioh etwa $1 \frac{1}{2}$ Jahre nach dem die Krankheit herbeifuhrenden Ereignis infolge einer neuen Geroütsbewegung eine unvollkommene Lähmung des linken Beines hinzugesellt. Der 4. Fall zeigte am linken Bein das Bild der Oppenheim'schen Akinesia amnestica, welche aber lange Zeit nach der Verwundung, gleichfalls infolge einer psychiscben Erregung, sekundär aufgetreten war. Der 5. Fall ist durch sehr hochgradige Zyanose, Oedem und Kälte der gipfelnden Teile des verletzten Beines ausgezeichnet, in welchem auch die Beweglichkeit erheblich herabgesetzt ist. Der 6. Fall endlich zeigte ätnliche Störungen in geringerem Grade nach einer einfachen Fussverstauchung.

Der Vortragende führt dann aus, dass die in dem zweiten der vorgestelltea Fälle so klar zutage liegende Entstehung der Erscheinungen zu der Annahme berechtige, dass auch die anderen Fälle psychogener Natur seien. Der eine der Fälle von schlaffer Monoplegie lege den Gedanken nahe, dass vielleicht in manchen Fällen traumatisch entstandene primäre lokale Angioneurosen das Material für die weitere psychische Verarbeitung lieferten. Das Wesentliche bleibe dabei aber immer der psychische Vorgang. Der affektrolle Zustand, den wir im Augenblick der Schädigung und mehr oder minder lange darüber hinausdauerad annehmen dürfen, schaffe einen guten Boden für das Haftenbleiben von fremdartigen Organgefüblen, die von irgend einer verletzten Körperstellé ausgelöst werden. Dieser Vorgang spiele sich entweder im Augenblicke der Schädigung selbst ab (traumatische Suggestion Charcot's) oder es lromme zur langsameren Ausarbeitung unter dem Einfluss der von dam Gliede dauernd ausgehenden Reize, wobei Anfmerksamkeit und Wunschtendenzen fixierend wirken.

(Eigenbericht.)

3. Herr Rosenfeld-Strassburg: „Ueber funktionelle Lähmungen bei Krjegsverletzten."

Vortragender demonstriert 5 Fälle mit funktionellen Extremitätenlähmungen bei Kriegsverletzten. Diesen Fällen ist gemeinsam: die schlaffe Armlähmung, die normale elektrische Erregbarkeit sämtlicher Muskeln für beide Stromarten, die mehr oder weniger ausgesprochenen angioneurotischen Störungen, eine leichte Abmagerung der gesamten Muskulatur ohne nennenswerte Atrophie der Knochen und der anfangs stets vorhandene Verlust des Organgefühls in der betrefienden Extremität, verbunden mit bandschulförmigen Anästhesien. Ferner ist beachtenswert, dass die funktionelle Lähmung sich stets auf der Seite der Verletzung findet. In sämtlichen Fällen fehlen neuropathische und psycho. pathische Antezedentien. Gleichgültig für die Entstehung, die Schwere und den Verlauf solcher Lähmungen sind: die Sebwere des Traumas, die Art und der Ort der Verletzung auf der gleichen Körperhälfte, die Intensität des psy- 
chischen Shocks und die Intensität der sogenannten mechanischen Erschütterung. Alle diese Faktoren können variieren und vollständig fehlen. Die psycbogene Natur der Störung tritt in vielen Fällen dentlich zutage und gibt sich zu orkennen in hysterischen Sensibilitätsstörungen, in raschen Remissionen in der Intensität der Lähmung, in psychisch bedingter Verschlechterung des Zustandes und in guter Realtion auf suggestive Therapie, auf Arbeitstherapie und Beschäftigung im Beruf. In manchen Fällen dominieren die scbweren angioneurotischen Störungen mit Verlust des Organgefühls in der betreffenden Extremität. Die angioneurotischen Störungen können zum Teil, wie z. B. die Oedembildung, sekundärer Natur sein und ihrerseits dann die Trophik des Arms und dadurch die Prognose des Falles ungünstig beeinflussen. Es ist aber nicht ohne weiteres von der Hand zu weisen, dass gewisse Funktionsänderungen im vasomotorischen System der betreffenden Extremität beim Zustandekommen der Organgefüblsstörung daselbst im Moment der Verletzung eine Rolle spielen, und somit ein wichtiges Glied in der Kette von Umständen darstellen, welche zu der Entstehung dieser funktionellen Lähmungen bei jungen Männern ohne nervöse Antezedentien fübren.

(Eigen bericht.)

4. Herr Mann-Manneim: "Ueber rasche Wiederkehr der motorischen Funktion nach Ulnarisdurchschneidungen mit lirankendemonstration."

Mann kerichtet über 9 Fälle von Ulnarisreseltion, die durch auffallend rascbe Wiederkehr der Motilität interessant sind. Er hebt ausdrücklich hervor, dass die Fälle nicht etwa als Operationsresultate gezeigt werden. sondern als Merkwürdigkeit, für die eine befriedigende Erklärung bisher fehlt.

Ungewöhnlich rasche Wiederkehr der motorischen Funktion ist auch von anderer Seite wiederholt berichtet worden. Vortragender erinnert an die in der Bardenheuer'schen Arbcit 1908 angefübrten Fälle, von denen fünf ebenfalls den Ulnaris betrafen, an den von Goldmann 1906 vorgestellten Fall von Resektion eines Stückes Ulnaris ohne Funlitionsausfall, an Fälle von Thiemann, Hobmann, Cassierer und Nonne. Bei den rom Vortragenden beobacbteten Verletzungen peripherer Nerven ist eine so rasche Wiederkehr nur beim Ulnaris gesehen worden. Es waren Fälle, bei denen die Verletzung schon mindestens drei Monate zurücklag, die diagnostisch sicher waren (Atrophion, trophiscbe Störungen, komplette Ea.R., sensible Störungen, die dem Versorgungsgebiet des Ulnaris entsprachen), und bei denen die Autopsie in viro einen dem klinischen Befund entsprechenden anatomischen ergảb. Die Fälle werden durch nachstehende Tabelle kurz erläutert; Fall 1 erinnert an den von Goldmann berichteten.

M. bespricht kurz die Erklärungsmöglichkeiten, die durchweg nicht voll befriedigen. Eine prima intentio hält $\mathbb{N}$. auch im Fall 1, der zunächst an eine solche erinnern könnte, für ausgeschlossen. Die Auswachsungstheorie, autogene Regeneration, Kombination von Auswachsung und autogener Regeneration, Kollateralbildung möglichter Anastomosen vom Medianus werden kurz kritisch angeführt. 
254 41. Wandervers. der Südwestdeutschen Neurologen u. frrenärzte.

\begin{tabular}{|c|c|c|c|c|c|}
\hline $\overrightarrow{\bar{z}}$ & Verwundung & Muskelbefund & Elektr. & Operation & $\begin{array}{l}\text { Wiederiblir } \\
\text { der Funktion }\end{array}$ \\
\hline 1 & $\begin{array}{c}17.3 .1915 \\
\text { Rechter Ober- } \\
\text { arm, unteres } \\
\text { Drittel. G. G. }\end{array}$ & $\begin{array}{l}\text { leichte Schwäche beim } \\
\text { Händedruck }\end{array}$ & $\begin{array}{l}\text { leichte galvanjsche } \\
\text { Zuckungsträgheit in } \\
\text { Hypothenar u. Inter- } \\
\text { ossei }\end{array}$ & $\begin{array}{l}111^{1 / 2} \text { Monate nach der } \\
\text { VerletzungResektion } \\
\text { eines Neuroms, } 2 \mathrm{~cm} \\
\text { lang }\end{array}$ & $\begin{array}{l}\text { Motorisoh. Be } \\
\text { fund ebens } \\
\text { gut wie ro } \\
\text { der Duroh } \\
\text { schneidung. }\end{array}$ \\
\hline 2 & $\begin{array}{c}\text { 27. 8. } 1914 \\
\text { Dicht oberbalb } \\
\text { d. Euenbogens } \\
\text { G. G. }\end{array}$ & $\begin{array}{l}\text { Lähmung des Eypo- } \\
\text { thenar, Jnterossei, } \\
\text { III und IV, Atropliie } \\
\text { der Interossei uud } \\
\text { Hypothenar }\end{array}$ & $\begin{array}{l}\text { kompl. Ea.R.im Hypo- } \\
\text { thenar u. Interossei } \\
\text { I, III, IV, nurober- } \\
\text { lialb der Jarbe } \\
\text { ansprechbar }\end{array}$ & $\begin{array}{l}10 \text { Monate nach der } \\
\text { Verletzung Narben- } \\
\text { reseltion, } 5 \text { om (Ner- } \\
\text { venenden bajonett- } \\
\text { artig in ier Narbe } \\
\text { ubereinander) }\end{array}$ & $\begin{array}{l}\text { 1. Stunde nac. } \\
\text { d. Opcration }\end{array}$ \\
\hline 3 & $\begin{array}{c}25.10 .191 j \\
3 \text { em oborbalb } \\
\text { d. Ellenbogens } \\
\text { G. G. }\end{array}$ & $\begin{array}{l}\text { Lähmung des Hypo- } \\
\text { thenar u. Interossei, } \\
\text { Atrophie }\end{array}$ & $\begin{array}{l}\text { kompl. Ea Pu.im Hypo- } \\
\text { thenar und Interossei }\end{array}$ & $\begin{array}{l}3 \text { Yonate nach der } \\
\text { Yerletzung Narben- } \\
\text { ezzision, } 2 \mathrm{~cm}\end{array}$ & $\begin{array}{l}\text { nach einigen } \\
\text { Stunden. }\end{array}$ \\
\hline 4 & $\begin{array}{l}\text { 10. } 2.1915 \\
\text { Oberarm } \\
\text { G. G. }\end{array}$ & $\begin{array}{l}\text { Lähmung der Inter- } \\
\text { ossei, d. Hypothenar, } \\
\text { Thenar, Parese der } \\
\text { Hand-u. Fingerbeug. }\end{array}$ & $\begin{array}{l}\text { kompl. sehwere Ea.R. } \\
\text { in allen Ulnar- } \\
\text { muskein }\end{array}$ & $\begin{array}{l}8 \text { Monatenach derVer- } \\
\text { letzung Naht nach } \\
\text { Durehtrennung }\end{array}$ & n. etra 8 Sti \\
\hline$\check{5}$ & $\begin{array}{l}\text { 28. } 1.1915 \\
\text { Obcrarm } \\
\text { G. G. }\end{array}$ & $\begin{array}{l}\text { Lahmung dos Hypo- } \\
\text { thenar u. Interossei, } \\
\text { Atrophie }\end{array}$ & $\begin{array}{l}\text { kompl. Fa.R. im Hypo- } \\
\text { thenar u. Interossei, } \\
\text { nuroborhalb dor } \\
\text { Narbe ansprech- } \\
\text { bar. }\end{array}$ & $\begin{array}{l}81 / 2 \text { Honate nach der } \\
\text { Verletzung Narben- } \\
\text { exzision, } 11 / 2 \mathrm{~cm}\end{array}$ & do. \\
\hline 6 & $\begin{array}{l}\text { 9. } 5.1915 \\
\text { Hoch am } \\
\text { Oberarm } \\
\text { G. G. }\end{array}$ & $\begin{array}{l}\text { Interossei, Hypothe- } \\
\text { nar,Thenar,Atrophie }\end{array}$ & $\begin{array}{l}\text { rompl. Ea.P. in Inter- } \\
\text { ossei, Hypothenar, } \\
\text { Thenar. Partielle } \\
\text { Ea.R. im Flex. carp. } \\
\text { ulnaris }\end{array}$ & $\begin{array}{l}9 \text { Monate nach der } \\
\text { Verletzung Narben- } \\
\text { exzision, } 2 \mathrm{~cm}\end{array}$ & n. etwa $24 \mathrm{Stc}$ \\
\hline 7 & $\begin{array}{l}\text { 21. } .1915 \\
\text { G. G. }\end{array}$ & $\begin{array}{l}\text { Flex. earp. uluaris, } \\
\text { Hypothenar, Inter- } \\
\text { ossei, Atrophie }\end{array}$ & $\begin{array}{l}\text { rompl, Ea.R. im Flex. } \\
\text { carp. ulnaris, Hypo- } \\
\text { thenar u. Interossei }\end{array}$ & $\begin{array}{l}6 \text { Monaìc nach der } \\
\text { Verletzung Narben- } \\
\text { exzision, } 21 / 2 \mathrm{~cm}\end{array}$ & $\begin{array}{l}\text { 3. Wochen nace } \\
\text { d. Operatior }\end{array}$ \\
\hline 8 & $\begin{array}{l}12.2 .1915 \\
\text { Oberarm }\end{array}$ & $\begin{array}{l}\text { Lähmung der Inter- } \\
\text { ossei u. Hypothenar, } \\
\text { Atrophie }\end{array}$ & $\begin{array}{l}\text { kompl. Ea.R. in den } \\
\text { gelähmten Muskeln }\end{array}$ & $\begin{array}{l}8 \text { Monate nach der } \\
\text { Verletzung Narben- } \\
\text { exzision, } 11^{\prime} / 2 \mathrm{~cm}\end{array}$ & n. 3 Wochen. \\
\hline 9 & 21.5 .1915 & do. & $\begin{array}{l}\text { kompl.Ea. R. im Hypo- } \\
\text { thenar u. Interossei, } \\
\text { Terv nur ober- } \\
\text { halb der Narbe } \\
\text { ansprechbar }\end{array}$ & $\begin{array}{l}101 / 2 \text { Monate naeh der } \\
\text { Verletzung Narbe } \\
2-3 \mathrm{~cm} \text { lang cx } \\
\text { zidiert }\end{array}$ & do. \\
\hline
\end{tabular}

Fall 1 obne Ausfall. - 5 folgende Fälle Wiederłehr inncrhalb 24 Stunden. - 3 Fälle innerhall 3 Wochen. -- 3 Fälle (3,6 und 9) nach anfänglicher Besserung leichte Verschlechterung. - 3 Fäll $(2,5$ und 9) elektr. nur oberhalb der Narbe ansprechbar.

Da die zur Verfügung stehende Zeit abgelanfen ist, werden die Fälle ausserhalb der Versammlung demonstriert.

(Eigenbericht.) 
5. Herr Lotmar-Bern: "Zur Lehre von den funktionollen Läh. mungen (mit Krankendemonstration)."

Bei einem 22jährigen Infanteristen ist im September 1915 nach halbjährigem Felddienst, obne Eiuwirkung von Granatexplosion oder dergl. und ohne äussere Verletzung, beim Marschieren Spannungsgefühl im linken IKnie, bald darauf stechender Schmerz im linken Hüftgelenk mit hochgradiger: Bewegungsbeschränkung und słarkem Hinken eingetreten. Im Lazarett kamen dazu Schmerzen auch in linker Iland und linkem Fuss, Verdickung derselben, Blauwerden beider Hände and Füsse, links überwiegend. Die Untersuchung ergibt jetzt: starker Bewegungsschmerz im linken Hüft- und Ellbogengelenk, geringerer im linken Hand- und Schultergelenk, Spannungsgefühl im linken. Kniegelenk, nirgends Knirschen oder Knacken, der Schmerzhaftigkeit entsprechender überwindbarer Muskelwiderstand bei passiver Bewegung; röntgenologisch leichte Atrophie der Gelenkkörper des linken Eillbogengelenks, negativer Befund am linken Hüftgelenk und Hand. Leichte, nicht wegdrückbare. Hautschwellung am linken Handrücken und Fingern sowie an linker Knöchelgegend; hochgradige Zyanose der linken Hand, des linken Knies und Fusses, viel geringere der entsprechenden Teile rechts. Ausgesprochene Parese des. ganzen linken Arms und Beins von funktionellem Charakter, eine sich ebenfalls. als funktionell kennzeichnende Empfindungsstörung links am Ober- und Unterarm, Rumpf, Ober- und Unterschenkel für Schmerz und Temperatur, etwas. weniger ausgebreitet für Berührung. Stark erhöhte Schweisssekretion links am. Rumpf und Bein, Steigerung der mechanischen Hautgefässerregbarkeit links am Rumpfe. Mässige diffuse Atrophie der Ober- und Unterschentel, wio der Armmuskeln links. Sehr schwacher Rachenreflex, sonst liein Hirnnervenbefund. Mässige Pulsbeschleunigung, sonst kein innerer Organbefund. - Da für die vorliegende Parese, Empfindungs-, Gefäss- und Schweissstörung ein organisches Nervenleiden (besonders Polyneuritis oder Syringomyelie oder zerebrale Hemi-plegie) sowie eine vasomotorisch-trophische Neurose (Acroasphyxia chronica) als Erklärung nicht in Betracht kommen, anderseits die vorhandene diffuse Muskelabmagerung und Gelenkatrophie die Auffassung des ganzen. Bildes als funktionell (Hysterie) schwierig machen, erscheint folgende Deutung als die wahrscheinlichste: infolge schmerzhafter organischer Erkrankung der Gelenke des linken Arms und Beins traten Bewegungsstörung, (reflektorische) Muskelund Gelenkatrophie auf. Zu der durch Schmerzen ausgelösten Schwäche, die sich weiterhin auf psychogenem Wege zu hochgradiger Parese steigerte, haben. sich dann ebenfalls auf psychogenem Wege die sensiblen, vasomotorischen und sekretorischen Störungen gesellt. Die Beschränkung der ursächlichen Gelenkaffektion selbst auf die linke Seite entzieht sich einer Erklärung. Bemerkenswert ist das Uebergreifen der Akrozyanose auf die sonst unbeteiligte rechte Seite. - Symptomatologisch besteht offensichtliob Verwandtschaft zu solohen (z. B. traumatischen) Gelenkerkrankungen, in welchen ausser der hä̀ufigen, jetzt. von den meisten als reflektorisch entstehend aufgefassten Muskel-, eventuell auch Knochenatrophie sowie lokalen vasomotorischen Störungen sich eine psychogene Empfindungsstörung findet. Vortragender sah vor kurzem einen Fall, 
der in dieser Weise aufgefasst, aber ohne Zwang auch nach Analogie des hier demonstrierten gedeutet werden konnte. Vielleicht ist jene symptomatologische Verwandtschaft eine mehr als äusserliche, doch wäre dies einer ausführlicheren Darlegung vorzubehalten.

(Eigenbericht.)

6. Herr Hoche-Freiburg: "Ueber die Frage der Dienstbeschädigung bei nervös und psyobisch orkrankten Feldzugsteilnehmern."

Der Vortragende erörtert die als Folgen nervöser und psychischer Kriegserkrankung zu erwartenden sozialen und finanziellon Schwierigkeiten, unter denen die Behandlung der traumatischen Kriegsneurosen an der Spitze stehen wird. Die Militär-Pensionsgesetze lassen von einem Einfluss der Friedenserfabrungen in der Unfallneurosen-Frage so gut wie nichts erkennen. Es ist aus verschiedenen Gründen, die näher auseinandergesetzt werden, mit Sicherheit zu erwarten, dass in zahlenmässig ungeheurem Massstabe Ansprüche aus tatsächlichen oder angeblichen nervösen Kriegsfolgen zur Anmeldung kommen werden. Der nach den allgemeinen bürgerlichen Erlebnissen beste Weg der Srledigung solcher Ansprüche, der endgültigen Abfindung mit kleinen Kapitalsummen, ist durch die jetzige Pensionsgesetzgebung ausgeschlossen. Das rom Reichstag angenommene Kapitalabfindungsgesetz beziebt sich nur auf Fälle mit stabilem Befunde, kapitalisiert in schematischer Weise nur einen Teil der Bezüge und ist nach allen Richtungen für das von uns zu erstrebende Ziel unwirksam. In Anbetracht der zweifellosen Tatsache, dass nur auf dem Wege der Kapitalabfindung eine schwere Schädigung der allgemeinen Volksgesundheit und Arbeitsfähigkeit durch Kriegsneurosen verhütet werden kann, erwächst den Nervenärzten die verantwortungsvolle Aufgabe, mit allen Mitleln nach einer Aenderung der bestehenden Pensionsgesetze zu streben. Der Vortragendo berichtet über die von ibm bisher in dieser Angelegenheit unternommenen Schritte und erbittet das Interesse und die Mitwirkung der Versammlung, damit möglichst im Herbst dem Reichstag eine Norelle zum Kapitalabfindungsgesetz, die den Bedürfnissen der Sachlage auf dem Gebiete der Nenrosen gerecht wird, porgelegt werden kann.

(Eigenbericht.)

7. Herr Hauptmann-Freiburg/Br.: „Die "Anfällo" der Kriegsteilnebmer."

Die Uebersicht über ein reichliches einschlägiges Material in der militärischen Beobachtungs-Station für Nervenkranke fübrte zur Aufstellung folgender 3 Fragen:

1. Gibt es charakteristische Anfallssymptome, aus welohen allein die Differentialdiagnose zwischen Epilepsie und Hysterie gestellt werden kann?

2. Gibt es eine „Kriegs-Epilepsie", d. b. eine Epilepsie, die bei bis dahin intaktem Nervensystem allein auf die somatischen und psychischen schädigenden Momente des Felddienstes zurückzufübren ist? (Posttraumatische Epilepsie ist hierbei nicht berücksichtigt). 
3. Kann man in der Gruppe der psychogenen Anfälle Untergruppen unterscheiden, oder ist alles der Hysterie zu subsumieren?

Unbedingt gültige Einzelsymptome im Anfall gibt es nicht (Zungenbisse, Pupillenträgheit, Urinabgang wurde auch bei psychogenen Anfällen beobachtet).

Der Nachweis irgend welcher somatischer hysterischer Störungen ist nicht unter allen Umständen ausschlaggebond für die Genese der betreffenden Anfälle (Vorkommen hysterischer Störungen bei sicherer Epilepsie, Vorkommen von hysterischen und epileptischen Anfällen am gleichen Individuum, was aber nicht "Hystero-Epilepsie" genannt werden darf).

In ca $90 \mathrm{pCt}$. finden sich bei den Patienten mit psychogenen Anfällen erblich belastende Momente und degeneratire Stigmata in der Vorgeschichte.

Eine Kriegs-Epilepsie gibt es nicht. In 99 pCt. fand Hauptmann hereditäre Belastung, degenerative Stigmata oder Anzeichen für das Vorliegen einer epileptischen Anlage, woraus geschlossen werden kann, dass nur ein von vornberein irgendwie prädisponiertes Gehirn durch die körperlichen und seelischen Schädigungen des Felddienstes zu einer epileptischen Aeusserung veranlasst werden kann. - Die Annahme einer Dienstbeschädigung wird dadurch natürlich nicht berührt.

Sehr charakteristisch ist die Unabhängigkeit des Auftretens der epiIeptischen Anfälle von emotionellen Momenten. Ebenso bemerkenswert ist die besondere „Kriegsfreudigkeil" der Epileptiker, beides so konstante Erscheinungen, dass sie differentiell-diagnostisch verwertet werden können.

Unter den psychogenen Anfällen sind 3 Gruppen zu trennen:

1. Hysterische Anfälle, weniger durch den Ablauf des Anfalls selbst charakterisiert, als durch das psychische bysterische Verbalten, den "Willen zur Krankheit", der sich auch in dem gewissermassen demonstrativen Charakter des Auftretens des Anfalls bemerkbar macht.

2. "Reaktiv-psychogene" Anfälle, d. h. solche, die, nachdem einmal durch ein emotionelles Moment (Granatshock etc.) der Weg des Anfalls gebahnt war, an den Patienten, auch gegen ihren Willen, infolge ihrer gesteigerten Reagibilität ablaufen, wenn ein neuer emotiver Reiz sie trifft.

3. Psychasthenische Anfälle, für deren Entstehen erschöpfende Momente (bei vorhandener Anlage) eine Rolle spielen. Vasomotoriker.

(Erscheint ausführlich an anderer Stelle).

(Eigenbericht.)

8. Herr Kaufmann-Ludwigshafen: "Die Ueberrumpelungsbehandlung der psychogenen motorischen Reizerscheinungen bei Soldaten."

Kaufmann hält es für notwendig, die psychogenen motorischen Reizerscheinungen bei Soldaten zum Sohwinden zu bringen, wenn dieselben die bei Feldzugsteilnehmern so gut wie nie fehlenden Erscheinungen der akuten 
Neurasthenie überdauern. Er bedient sich zu diesem $Z$ wecke nach entsprechender suggestiver Vorbereitung der Wachsuggestivmethode unter Zuhilfenahme kräftiger, unter Umständen schmerzhafter Wechselströme, unter Ausnutzung des militärischen Vorgesetztenverhältnisses: Frteilen der Suggestionen in schärfster Befehlsform unter Zuhilfenahme der militärischen Kommandoworte. Das Geheimnis des Erfolges liegt in der unerschütterlich konsequenten Durchführung der Behandlung in einor Sitzung. Psychogene Tremores und Ties bringt man auf diese Weise oft in wenigen Minuten zum Schwinden. Es kommt vor, dass die Störung zunächst zunimmt; das darf aber nicht veranlassen, dio Sitzung zu unterbrechen. In vereinzelten sehr komplizierten Fällen hat Vortragender bis zu mebreren Stunden gebraucht.

Geheilt wurden auf diese Wsise eine ganze Reibe von Tremores, Ties, spastischen und schlaffen Lähmungen, die verschiedenartigsten psychogenen Gehstörungen, Matistische, Taubstumme, Aphoniker. - Kein Erfolg wurde erzielt bei Stottern und Tachypnoe.

Besonders indiziert ist das beschriebene Vorgehen bei verschleppten Fällen. Eine Kontraindikation bildet die Komplikation mit schweren Erscheinungen akuter Neurasthenie, mit hochgradigem Erethismus, mit sehr ausgesprochener explosiver Diathese.

(Eigenbericht.)

9. Herr Kehrer-Freiburg/Br, z. Z. Res.-Laz. Bärenstein: „Ueber Entstebung und Behandlung der Kriegsneurosen."

Kehrer stellt den erfreulichen Offensivgeist fest, der sich im Verlaufe des abgelaufenen Jahres seit der letzten Versammlung besonders im Bereiche des XIV. Armeekorps in der Behandlung der Kriegsneurotiker geltend gemacht hat. Er berichtet von den Erfahrungen, die er seit Herbst 1915 als Leiter des 100 bettigen „Funltionellen-Lazaretts" Bärenstein (bei Bühl, Höhe $800 \mathrm{~m}$ ) gewonnen hat. Als das Wichtigste erscheint ihm das Durchsetzen der militärischen Autorität bis in alle Einzelbeiten. Bei richtiger Unterbringung und Verteilung ist die Gefahr psychischer Infektion und Indultion fast gleich Noll. Gelegentliche gerechte Disziplinierung ist besonders bei den "Verziehungshysterikern", „psychopathisch Minderwertigen mit hysterischen Allüren, besonders den als Vaterlandsverteidiger" sich Aufspielenden, auch wenn Schreck- oder GranatShock sie dienstunfähig gemacht hat, von heilsamer Wirkung. Seitdem Kehrer zur Propbylaxe grosser hysterischer Anfälle ev. regelmässig abendliche feuchte Ganzpackungen ausführt, hat er trotz Häufung der dazu Disponierenden nur mehr selten solche zu sehen bekommen. Zur Beseitigung der sinnfälligen funktionellen Reiz- und Lähmungserscheinungen ist zulässig und brauchbar jede Methode; es muss nur der nach der individuellen Färbung des Falles vorher modifizierte Heilungsentwurf mit aller durch ärztliche Ethik zu rechtfertigenden Strenge und unter Heranziehung aller Register militärischer und pädagogischer Autorität rücksichtstos bis zu Ende durchgeführt werden. Behandler und Behandelter müssen gleicher Weise in der Methode ,aufgehen". Die ,kleinen" Methoden auch inbezug auf das Anwendungsgebiet sind: Selbstausschaltung des Kranken aus seinem geistigen bzw. lrameradschaftlichen Interessenkreisè 
durch die Folgen des Hauplsymptoms ("Zutodelangeweilen "); Einschaltung eines mehrwöehigen Urlaubs nach Vergewisserung über günstige psychologische Struktur des fariliären Kreises (Ehefrau, Brant) besonders bei kosmetisch unangenehm auffallenden Reizsymptomen; rationale Methode nach Dubois für die Stadien, in denen "Phobie ohrie Fremdheitsgefühl" bei dem Versuche der Symptomenüberwindung diese versperrt; Methode der Mobilisierung verdrängter oder (bildlich!), ,sekundär atrophisch" gewordener Bewegungsvorstellungen durch geduldig-strenges Wiedererlernenlassen der richtigen Innervationsverteilung. Die grossen" Methoden sind: Die Hypnose und die ron ihm besonders ausgeübte "Gewalt-Exerzierkur" (Exerzier-Reglement!). Kehrer vergleicht sie mit der Methode, die ein Reitkünstler anwenden muss, um ein durch schlechte Reiter an zahllose Unarten gewöhntes, "gutes" Reitpferd durch genaue Einfühlung und Dosierung aller "Hilfen" wieder in die korrekten Gangarten zu bringen. Dem Sporn würde der schmerzhafte elektrische Strom entsprechen, dessen Anwendbarkelt sich von Fall zu Fall ergibi, bei Analgesien unentbehrlich, bei misstrauisch-schüchternen Debilen gegenangezeigt ist. Sehr viel kommt auf die richtige Einschätzung des situativen Seelenzustandes bzw. der Persönlichkeit an. Natürlich zielt auch diese Methode nur darauf ab, sinnfällige Symptome aus der Dauerform in die "unschädliche Latenzform" zu überführen. Anwendungsgebiet ist: Alles Funktionelle; ausser der anerkannten Hysterie alle "Oppenheim-Neurosen" und alle Fälle mit der" Etikette: Simulation oder Hysterie! Ausgeschlossen sind alle sicher organischen Erkrankungen, die echte nervöse Erschöpfung und die Organneurosen. Innerhalb jenes Gebiets sind so heilbar alle sinnfälligen Reiz- oder Ausfallerscheinungen an den Erfolgapparaten des spinozerebralen Systems, weitgehend beeinflussbar alle vorwiegend, autonomen Symptome". Kehrer verfügt bereits über $3 \mathrm{Fälle}$, in denen schwere Oedeme der distalen Armabschnitte 12--24 Stunden nach der Heilung der "Akinesia amnestica" bzw. "Reflexlähmung", über einen, in dem Hyperhidrosis und einwandfrei festgestelltes Fieber bei Schüttelneurose verschwanden und in Wochen nioht wiederkehrten; über wieder andere, in denen die kardiovaskulären Anfälle beseitigt wurden. Kehrer liommt zu dem Ergebnis, dass in bezug auf psychische Beeinflussbarkeit zwischen Hysterie und "Oppenheim-Neurosen" nicht der geringste Unterschied besteht. Obwohl Kehrer die Häufigkeit von vorwiegend "autonomen Störungen " (vasovegetativen, trophischen, hidrotischen, kardiovaskulären etc.) bei Kriegsueurotikern besonders unterstreicht, spezielle Dispositionen für Zyanose, Oedem usf: für gewisse Fälle sogar postuliert, findet er doch, bei der Gegenprobe seines Materials, dass gerade die relativ geschlossensten Verbindungen von Symptomen dieser Kategorien bei Kriegsteilnebmern zu finden sind, die sich eines prononzierten Fronterlebnisses (Schreck, Granatshock usf.) nicht im geringsten entsinnen können.

Bei der Entstehung der Kriegsneurosen wirken psychische, psychophysische und rein körperlicho Momente in grösster Variabilität in-, auf- und darcheinander. Die Dehnbarkeit der psychisch-nervösen Disposition zeigt sich besonders an den "negativen Fällen", der nicht geringen Zahl yon endogen 
Nervösen verschiedenster Schattierung, besonders zu synkopalen, vagovasalen und ähnlichen Anfällen Disponierenden, die sogar nach sicherem Schreckoder Granatshock weder Zeichen von Hysterie noch "Oppenheim-Neurosen" zeigen. Fast eindeutig dagegen sind die Falitoren, welche die Ortswahl besonders der lateralisierten Reiz- oder Lähmungserscheinungen bestimmen. Es gibt kaum einen Fall, in dem diese nicht kausal oder aus verständlichem $\mathrm{Zu}$ sammenhang, entweder aus der psychischen bzw. reaktiven Ueberwertigkeit des befallenen Körperabschnitts besonders beim „Trauma" oder im Stadium der Restitution der tramatischen Bewusstlosigkeit oder aus einer irgendwie gearteten, - nervösen oder nicht nervösen, alten oder frischen - dynamischen Minderwertigkeit dieses Körperteils erklärbar wäre. So sehr Kehrer auf die rein körperliche Grundlage vieler neurotischer Symptome Wert legt, von dieser Seite her sind sie nicht, vielmehr nur von der psychischen her, zu beseitigen. Mit Bezug auf die einfache Schreck- oder Granatshockwirliung hält Kehrer die Formulierung für gerechtfertigt: Es gibt keine Stärke eines einmal auf dem gewöhnlichen Sinneswege das Gehirn treffenden Ueber-Reizes, deren sinnfällige Folgen bei vorher gesundem Organismus auf psychischem Wege nicht ausgleichbar wären, vorausgesetzt, dass nicht die Perzeptionsapparate des betreffenden Sinns selbst durch den physikalischen Reiz lädiert worden sind.

(Eigenberieht.)

10. Herr Schmidt-Freiburg: „Ueber psychischen Puerilismus bei Kriegsteilnehmern."

Der Vortragende berichtete über 2 Fälle von Puərilismus im Sinne von Dupré, Charpentier, Courbon und Sträussler. Das Syndrom des Puerilismus ist ein kleiner Ausschnitt aus dem polymorphen Bilde der Störungen nach Shockerlebnissen wie Granatexplosionen und Minenverschüttungen; es weist jedoch neben zahlreichen anderen klinischen Erscheinungen auf den Parallelismus hin, der die haftpsychatischen Erscheinungen mit den Störungen nach Shockerlebnissen im Felde verbindet.

(Eigenbericbt.)

11. Herr Schultze-Bonn: „Bemerkungen über traumatische Neurosen, Neurasthenie und Hysterie."

Der Vortragende nimmt Bezug auf seinen vor 27 Jahren gehaltenen kurzen Vortrag über die sogenannte "traumatische Neurose" auf der XIV. Wanderversammlung der südwestdeutschen Neurologen und Irrenärzte. Mit den damaligen Bemerkungen wurde die grosse Reihe der späteren Besprechungen des gleichen Gegenstandes eröffnet. Es wird ein kurzer Ueberblick über die historische Entwicklung der damals berührten Fragen gegeben, besonders über die etwaige Besonderheit einer nur nach Traumen sich entwickelnden Neurose, sodann über die Bedeutung der konzentrischen Einengung des Gesichtsfeldes und endlich über die Häufigkeit der Simulation und Uebertreibung.

Sodann wird über die Weiterentwicklung der Krankheitsbilder der Hypochondrie, Neurasthenie und Hysterie gesproehen. Der letzteren wird nach der Ansicht des Vortragenden jetzt von vielen ein viel zu breiter Raum zugewiesen; 
die Neurasthenie müsse in sekundäre und primäre unterschieden werden. Sie seien keineswegs stets psychogener Natur, wenn auch von manchen leichte echt psychotische Zustände fälschlicherweise als Neurasthenie bezeichnet werden.

Dem neuerdings von 0 p penheim gezeichneten Bilde der traumatischen Neurose im engeren Sinne fehle das Bild eines einheitlich gestalteten Symptomenkomplexes, wenn auch durchaus anzuerkennen sei, dass nicht alle solche Symptomenkomplexe stets psychogener Natur seien. Man müsse auch z. B. direkte Kommotionsstörungen zulassen, die nichts mit Hysterie zu tun hätten, die als Neurasthenien, oder einfach als funktionelle Lähmungen oder Krampfzustände zu bezeichnen seien. GleicheStörungen kämèn aber auch nach anderen als mechanogenen Einwirkungen vor.

Gegenüber der traumatischen Reflexlähmung verhält sich der Vortragende ablehnend, schon weil es ihm nicht angebracht erscheint, eine derartige hypothetische Annahme in eine Krankheitsbenennung hineinzunehmen.

Von einzelnen hysterischen Krankheitsersoheinungen wurde noch kurz auf das blaue Oedem Charcot's und auf das hysterische Fieber eingegangen. (Eigenbericht.)

12. Herr Westphal-Bonn: „Ueber einen Fall von ungewöhnlicher hysterischer Kontraktur."

Der Kriegsfreiwillige S. wurde durch einen Granateinschlag im Schützengraben verschüttet. Keine schwereren Verletzungen, nur einige schmerzhafte Kontusionen besonders des linken Unterschenkels und Fusses. Während der Behandlung in verschiedenen Lazaretten entwickelte sich die eigenartige Stellungsanomalie des linken Unterschenkels und Fusses, wegen deren Patient in unsere Klinik überführt wurde. Es handelte sich um eine Auswärtsrotation des linken Fusses um $180^{\circ}$, so dass bei parallel gerichteten Füssen der linke Hacken nach vorn, die Zehen nach hinten standen. Die linke Wade war dabei nach vorn, das Kniegelenk nach aussen gedreht. (Demonstration der Photographien). Im Bett lag der linke Fuss völlig nach aussen rotiert bei leicht gebeugtem Knie mit seiner äusseren Kante der Unterlage auf. Die Muskulatur des Unterschenkels fühlte sich gespannt an, schon bei leichtem Druck traten Schmerzen auf. Ausserhalb des Bettes beim Stehen und Gehen trat sofort wieder die beschriebene abnorme Stellung des Fusses ein. Pat. vermochte trotz dieser hochgradigen Verdrehung des linken Unterschenkels und Fusses flott zu gehen, verlangte wieder an die Front zurück. Der Gang des Pat. mit nach vorn gerichtetem Hacken und nach binten gedrebten Zehen bot ein recht groteskes Bild dar. Der Versuch, passiv die abnorme Fussstellung zu redressieren, rief die lebhaftesten Schmerzäusserungen hervor und misslang vollständig. In den betroffenen Muskelgruppen waren häufig neben fibrillären Zuckungen ausgiebige klonische Krämpfe gröberer Muskelbündel, die als sehr schmerzhaft bezeichnet wurden, zu konstatieren. Es bestand am linken Fuss and Unterschenkel eine strumpfförmige anästhetische Zone, bis etwas über das Ḱnie heraufreichend. Bis auf leichte vasomotorische Störungen (Tachykardie) im übrigen normaler Befund, insbesondere liess die Röntgenuntersuchung an den 
Gelenken und Knochen keine Veränderungen erkennen. Psychisch etwas theatralisches Wesen, lebbafter Stimmungswechsel und abnorme Reizbarkeit.

Die Behandlung war sehr langwierig und stiess auf grosse Schwieriglieiten. Hypnose-Versuche, elektrische Behandlung versagten völlig. Es gelang schliesslich nach Richtigstellung des Fusses in leichter Narkose, durch Streckverbände (Gipsverbände), die unter energischster Suggestion angelegt wurden, die Störung ganz allmählich zu beseitigen, so dass Pat. als garnisondienstfähig entlassen werden konnte und z. Z. noch garnisondienstfähig ist.

Wir gehen wohl nicht fehl in der Annahme, dass es sich hier um einen recht ungewöhulieben Kontrakturzustand von den Fuss nach aussen drehenden Muskelgruppen gehandelt hat. Auf den Reizzustand wiesen die erwähnten schmerzhaften Muskelzuckungen, die besonders lebhaft im Stadium des Nachlassens der Kontraktur in die Erscheinung traten, mit Deutlichkeit hin. Der Beginn der Stellungsanomalie des Fusses steht vielleicht mit $A$ bwehrbewegungen desselben gegen die durch die Kontusionen hervorgerufenen Schmerzen, und eine durch diese bedingte "Zwangshaltung" im Zusammenhang, welche dann im Laufe der Behandlung durch autosuggestive Einflüsse hochgradig gesteigert und in der abnormen Stellung fixiert wurde. Auf die psychogene Grundlage der Störung wiesen die anästhetische Zone, das hysterische Wesen des Pat., sowie die vorwiegend anf suggestivem Wege erzielte Heilung hin.

Bemerkenswerterweise konnte Pat. nach seiner Wiederberstellang die abnorme Stellung des Fusses in keiner Weise mehr hervorbringen; ich hebo diesen Umstand besonders hervor, da auch unter physiologisehen Verhältnissen bei grosser Uebung äbnliche bizarre Fussstellungen und groteske Gangarten wohl hervorgebracht werden können, wie man es mitunter bei Vorführungen von Clowns und Parterrealsrobaten zu beobachten Gelegenheit hat. (Eigenbericht.)

13. Herr Ph. Jolly-Halle/S., z. Z. Nürnberg: „Ueber Kriegsinvalidenfürsorge für Nervenkranke."

Es empfiehlt sich nicht, die dienstunbrauchbar werdenden Nervenkranken in eigenen Lazaretten zu vereinigen, weil dieselben ungünstig aufeinander einwirken würden, und weil es öfter für den Kranken recht gut ist, wenn er nicht weiss, ob er wieder Dienst machen muss oder nicht; ebensowenig ist es angebracht, die invalide werdenden unter die andern Invaliden zu mischen, da die richtige Beurteilung der Nervenkranken Sache des Spezialisten ist, und ihre Behandlung eine gewisse Gleichartigkeit verlangt, wie z. B. Alkoholabstinenz notwendig ist; auch würden Lente mit Krampfanfällen andere Kranke sehr stören, Hysteriker würden an chirurgischen Kranken viel Nachahmenswertes finden. Man sollte also wie bisher die Nervenkranken ohne Rücksicht anf ihre spätere Dienstfähigkeit im Lazarett belassen.

Dí Ziele der Kriegsinvalidenfürsorge für dieselben sind möglichste Besserung des Zustands, Wiedergewöhnung an Arbeit und Unterbringung am besten im alten Beruf. Für die ersten beiden Punlte ist ein wichtiger Falitor Beschäftigungs - und Arbeitstherapie im Lazarett. In dem meiner Leitung unterstehenden Lazarett mit 200 Betten haben wir nach langen Ver- 
handlungen auf Kosten der städtischen Kriegsivalidenfürsorge Nürnberg die folgenden Einrichtungen getroffen, in denen unter Aufsicht von Sachverständigen gearbeitet wird: Ein Zeichensaal fär Bauhandwerker (Maurer, Tischler, Schlosser etc.), eine Werkstätte für Kunstgewerbe (Maler, Lackierer, Bildhauer, Friseure etc.), eine Werkstätte für Tischler und eine solche für Metallgewerbe, Arbeitsgelegenheit für Schuster und Schneider, Unterrichtskurse für Kaufleute and Gewerbetreibende; ferner ist noch die Anstellung eines Hilfsschullebrers für Leute mit Schädelschüssen und für allgemeinen Volksschulunterricht beantragt. Dass für Landwirte keine Arbeitsgelegenheit besteht, ist kein sehr grosser Mangel, da dieselben meist sehr nach Hause drängen, auf dem Land gebraucht werden und sieh leicht in ibrem Beruf wieder zorechifinden.

Nach den Erfabrungen an solohen Patienten, die schon seit langem dio Schulungseiurichtungen in der Stadt besuchten, und an den jetzigen Patienten lässt sich sagen, dass der Eifer der Leute meist wider Erwarten gross ist, besonders da nur eine praktische, für den Beruf zu verwertende Tätigkeit ansgeübt, und möglichst durch Stellung besonderer Aufgaben das Interesse des Einzelnen geweckt wird. Besonders gute Erfolge haben wir bei den Verschlim. merungen der endogenen Neurasthenie und den Erschöpfungszuständen. Von diesen wurden viele wieder dienstfäbig.

Von den Hysterikern ist ein kleiner Teil nicht zur Arbeit im Lazarett zu bringen. Auffallend wenig stört nicht allzu hochgradiges Zittern, was für die Beurteilung der Erwerbsfähigkeit wichtig ist. Krampfanfälle blieben öfter ganz aus. Bei Zwangshaltungen und Lähnungen hysterischer Natur war der Erfolg gering.

Leute mit organischen Lähmungen lernten die gebliebenen Fähigkeiten gut auszunützen und sich wieder an den Gedanken der Arbeit zu gewöhnen.

Psychopathen und Imbezille nahmen kaum an den II ursen teil, da dieseiben nach ganz kurzer Zeit wieder entlassen wurden.

Zur Invalidenfürsorge gehört ferner besonders die Berufsberatung und die Unterbringung im Beruf. Letztere ist Sache der gemeindlichen, staatlichen und anderen Einrichtungen, während bei ersteren auch der Arzt ein Wort mitzusprechen hat. In letzter Zeit war ich bei der Berufsberatung in unserem Lazarett regelmässig dabei. Man erfährt dabei manche wichtige Einzelheiten über praktische Folgen der Erkrankung und den Beruf des Patienten und kann so dessen Erwerbsfähigkeit gerechter einschätzen, andererseits kann man die bäufgen Uebertreibungen der Patienten gegenüber dem Berufsberater richtigstellen. Praktisch am meisten geschädigt von unseren Kranken sind um nur einiges zu erwähnen - Leute mit Schädelschüssen und epileptischen Krampfanfällen, da dieselben wegen der Krämpfe von maschinellen Betrieben arsgeschlossen werden und nicht in der Höhe arbeiten können, ausserdem durch die zerebralen Allgemeinerscheinungen wie Kopfschmerzen und Schwindel bein Bücken ganz abgesehen von besonderen Herderscheinungen sehr gestört sind. Auch die Leute mit bysterischen Krampfanfällen sind wegen derselben in der Möglicbkeit, eine Steliung zu finden, oft erbeblich beeinträchtigt, während die Inteligenten unter den Invaliden häufig früher in ihrer Ausbildung Ver- 
säumtes nachbolen können, z. B. die Meisterprüfung nachmachen und sich öfter mit Vorteil spezialisieren, so statt Banführer, der auf dem Bau herumklettert, wegen Schwindelneigung Bauzeichner werden, ist es besonders schwierig, etwas für $D$ ebile zu finden. Letztere sind oft ungelernte Arbeiter und sind eigentlich relativ mehr geschädigt, wie ihre Schicksalsgenossen mit guter Intelligenz. Gerade diese etwas minderwertigen Elemente, aber auch manche ganz inteiiigente, jedoch wenig arbeitsfreadige Lente drängen sebr zu den sogenannten Verlegenheitsberufen der Portiers, Ausgeher und dergleichen, auch Schreiber wollen sehr viele Leute werden. Wenn die Leute nicht auf die Ratschläge hören, und wenn ihnen der Wille zu ernsthafter Arbeit fehlt, so kann man sie nur dem Daseinskampf überlassen.

Es ist sehr zu bedavern, dass für die funktionellen Neurosen noch nicht die Möglichkeit einer einmaligen Abfindung besteht, da voraussichtlich durch die Rente viele ron diesen Leuten für die Dauer verdorben werden. Die von manchen Stellen gehoffte wesentliche Besserung duroh Austritt aus dem Militärverband und Beendigung des Kriegs dürfte nur im beschränkten Masse eintreten.

(Eigenbericht.)

14. Herr G. Voss-Düsseldorf: "Erfahrungen über Simulation bei Militärpersonen."

Im Gegensatz zu der Meinung der meisten Fachleute, dass Vortäuschung und Uebertreibung bei unseren Soldaten selten seien, wird ihr Vorliegen von Laien (Vorgesetzten), aber auch von Aerzten verhältnismässig häufig angenommen. Vortragender hat aus dem grossen Material der als Beobachtungsstationen dienenden Cölner psychiatrischen Klinik und Militär-Nervenklinik des VII. A. K. in Crefeld eine Reihe von Fällen zùsammengestellt und geprüft. Die wegen Simulationsverdachtes Eingelieferten setzen sich aus Psychosen, Neurosen, organischen Krankheiten und Mischfällen zusammen. An Beispielen werden kurz die einzelnen Gruppen erläutert. Die Beurteilung echter Psychosen ist am einfachsten, schwieriger gestaltet sie sich bei Grenzzuständen (Psychopathen, Pseudologisten etc.). Auffällig war die grosse Zahl der Schädelverletzungen unter den Verdächtigen: sie bieten trotz schweren organischen Störungen, Fremdkörpern, psychischen Defekten bei viol subjelitiven Bescbwerden der oberflächlichen Untersuchung nur wenig objektive Anhaltspunke. Nach unserer Erfahrung ist diesen dauernd schwer Geschädigten gegenüber besondere Vorsicht in der kränkenden Annahme von Uebertreibung geboten. Zusammenfassend darf wohl gesagt werden, dass reine Simulation unter unserm Material sehr selten war; bäufiger kommt Aggravation vor, sowohl bei organisch Nervenkranken, als auch bei Neurosen. Krankhafte Uebertreibung liegtoftgonug in Wesen der klagsüchtigen Neurastheniker und vieler Hysterischon, sie muss von zweckbewusster Aggravation möglichst unterscbieden werden. Die Schwierigkeit dieser Unterscheidung kann gross sein, sie erfordert eingehende Untersuchung mit Berücksichtignng des psychischen Befundes. Vor allem erscheint eine Zusammenarbeit der verschiedenen Fachleute (Chirurgen, Internisten and Nervenärzte) bei der Beurteilung dieser Fälle geboten, nm 
unseren oft so schwer geschädigten Patienten den kränkenden Verdacht der Uebertreibung oder Simulation zu ersparen.

(Eigenbericht.)

15. Herr Dinkler-Aachen: „Ueber Insuffizienz der Wirbelsäule (Schanz) und ähnliche Krankheitsbilder auf kriegshysteriseber Grundlage."

Dinkler berichtet über das von Sobanz gezeichnete Bild der Wirbelsäuleninsuffizienz; die charakteristischen Erscheinungen: Schmerzen in den Bauch- und Brustorganen ohne organische Grundlage, verbunden mit einem ausgesprochenen Druck- oder Klopfschmerz der Dornfortsätze der mittleren Brust- oder der Lendenwirbel. 1910 hat Zuelzer an die Beobachtungen Schanz' angeknüpft und iu Gegensatz zu Schanz die Forderung aufgestellt, dass nur diejenigen Krankheitsfälle, in denen ausser den obengenannten Erscheinungen eine Deformierung der Wirbelsäule nachweisbar sei, als echte Wirbelsäuleninsufizienzen zu bezeichnen sind; die Bronchialdrüsenentzündungen mit ihren Folgen und die gelenkrbeumatische Erkrankung der Wirbelsäule sind streng abzutrennen. Zuelzer erwähnt einen typischen Insuffizienz-Fall von kypho-skoliotiseher Verkrümmung der Wirbelsäule mit Schmerzen im Leib, die zu einer zweimaligen Gallensteinoperation führten. Solche Fälle sind bei einiger Ueberlegung leicht za beurteilen. Schwieriger siad die typiscben Fälle ron Wirbelsäuleninsuffizjenz obne erkennbare Veränderung an den Wirbeln; folgender Fall mag zur Klarstellung des klinischen Bildes genügen: Bei einem 38jährigen Kaufmann treten Schmerzen im Lendenteil der Wirbelsäule am Ende des langen Arbeitstages auf, die in die Weichen ausstrahlen und bei Bettruhe sofort verschwinden; allmählich beginnen die Schmerzen schon nachmittags und mittags, dauern nachts längere Zeit an und erstrecken sich scbliesslich bei Fortdauer der Arbeit in stehender Stellung auf den ganzen Tag und die Nacht; bei der Untersuchung ist die Lendenwirbelsäule ausserordentich druck- und klopfschmerzbaft, die Haltung ist steif und unbeweglich; jede Behandlung (elektrische, Wasser-, Massage- etc.) bleibt erfolglos. Schmerzfreiheit und Heilung tritt ein, wenn die Wirbelsäule durch Liegen im Gipsbett entlastet und ruhig gestellt wird und später ein Stützkorsett längere Zett getragen wird. Die Schmerzen entsprechen den sogen. Wurzelschmerzen und sind verständlich, wenn man eine Art Erweichung bestimmter Wirbel und eine dadurch bedingte Kompression der durchtretenden Wurzeln annimmt. Mit der Streckung der Wirbelsäule hören die Druckerscheinungen auf, und nach längerer Zeit nehmen die entlasteten Wirbel wieder normale Festigkeit an, so dass das Korsett wieder weggelassen werden kann. Nach den Erfabrungen im Kriege scheint das Krankheitsbild der Wirbelinsuffizienz auch akut im Anschluss an einen mit einer starken Stauchung der Wirbelsänle verbundenen Unfall (beim Springen mit dem Pferde etc.) vorzukommen. Bei letzteren Fällen ist grösste Vorsicht erforderlich, um Verwechselungen mit hysterischen Störungen zu vermeiden; werden hysterische Haltungsstörungen mit Gipsbett behandelt, so sind die Folgen in therapeatischer Beziehung sehr unerfreulich.

Nähere Mitteilung erfolgt in der Münch. med. Wochenschrift. 
Diskussion zu den vorausgegangenen Vorträgen:

Herr Bethe-Frankfurt a/M. (zu Mann's Vortrag): Bethe hält eine Erklärung der schnellen Restitution nach Nervennahl auf Grund präexistenter Anastomosen rom Medianus zum Ulnaris für wahrscheinlich. Durch die Operation werden Hemmungen aufgehoben, welche vorher die Funktion der Anastomosen mehr odex weniger behinderten. Dafür spricht, dass auch vorher die Lähmung im Ulnarisgebiet meist nicht vollständig war, und dass die Kraft und der Ernährungszustand der betroffenen Muskeln seit der Operation kaum Fortschritte gemacht baben. Eine prima intentio hält Bethe nach dem Erfolg des Tierexperiments für ganz ausgeschlossen.

Herr Edinger-Frankfurt a/M. (zu Mann's Vortrag): Ich halte es für wahrscheinlich, dass die Verschlechterung, die Herr Mann in einigen bereits geheilton Fällen wieder neu auftreten sah, auf Aufbrauch der noch schwachen Nerven duroh ihre relative Ueberfunktion beruht. Jedenfalis war diese Ursache in einem meiner Fälle nachweisbar, wo oin Mann nacb vollkommener Heilung einer Ulnarislähmung neu erlahmte, nachdem er, mit seiner neuen Kraft renommierend, einen Baum gefällt und zersägt hatte. Er ist später wieder genesen. Die Geheilten müssen geschont werden.

Herr Becker-Baden-Baden (zu Mann's Vortrag): Wenn die Befunde des Vortragenden vor der Operation jmmer ganz genau die vorhandenen Funktioneu feststellten, so legt die auffallende unmittelbare Wirkung der Operation die Vermutung nahe, dass von dem dabei entfernten besehädigten Nervenstück zentripetale Reize ausgingen, welche hemmend auf die intakten anastomotischen zentrifugalen Bahnen wirkten.

Herr Mann (Schlusswort): Eine elektrische Reizuug während der Operation ist nicht gemacht worden.

Die von Edinger angefübrte Erklärung für die Verschlechterung der Funktion nach anfänglich guter Funktion erseheint Mann sehr plausibel. Die Leute haben sich in den Lazarettwerkstätten vielleicht überanstrengt.

Von den Erklärungsversuchen hält Mann die Annahme von Anastomosen vom Medianus für das wahrscheinlichste; vielleicht verhält es sich so, dass durch die Lösung vom alten Zentrum eine Art Blockierung aufgehoben und eine volle Verbindung mit dem gesunden Medianus hergestellt wird.

Herr Nägeli-Tübingen: Die Kapitalabfindung hat sich bei meinen Nachuntersuchungen über die Endschicksale in der Schweiz durchans bewährt. Alle wirklichen Neurosen wurden gesund und haben nachher bei Volllohn jahraus jahrein gearbeitet.

Von Kapitalverschleuderung konnte ioh nichts finden, Keiner ist je später der Armenbehörde zur Last gefallen. Nur 5 haben später Wirtschaften gegründet, 3 davon gehen sehr gut.

Deshalb ist in der Schweiz heute die Kapitalabfindung gesetzlich eingeführt, unter der Auszahlung einer Summe, die einer gewissen Erwerbseinbusse für 2-3 Jahre, nie länger, entspricht. 
Bei der Simulationsfrage kommt es sehr auf die Definition des Begriffes an. Es ist psychologisch ganz begreiflich, dass erst auf ein bestimmtes Ereignis hin, nicht ohne jeden Anlass, Klagen vorgebracht werden. Solche sind dann meist tatsächlich vorhanden, aber unerheblich und würden die Arbeitsaufnabme nicht hindern. Der Entscheid, keine Erwerbseinbusse, reicht daher meist völlig aus, und lässt das Eingehen auf die Frage der Simulation, die sehr schwer za beweisen ist, als unnötig erscheinen.

In diesem Sinne ist Simulation von Erwerbseinbusse freilich nicht selten.

Die Symptome neurologischer Natur für die Unfallsneurosen sind heute alle hochgradig entwertet, vielleicht mit Ausnahme motorischer Störungen. Vasomotorische Erscheinungen an den gelähmten Gliedern sehe jch für sekundäre mechanische Folgen an, durch Ruhigstellung und Hängenlassen. Dafür zeugt das rasche Verschwinden bei suggestiver Heilung, das Feblen ähnlicher Bilder, wenn z. B. psychogene Trunkusparese vorliegt. Ebenfalls führen viel hochgradigere vasomotorische Erscheinungen bei Syringomyelie und bei spastiscber Obstipation (Vagotonie) nie zu P aresen, auch nicht bei jahrelanger Daner.

Die Alinesia amnestica und die Reflexlähmungen sind bildliche Vergleiche, keine Erklärungen, die wir als nicht das Wesen der Sache treffend zurückweisen müssen.

Herr Determann-Freiburg/Br.-St. Blasien (zu Hoche's Vortrag): Ich halte es für wichtig, dass, wie Hoche es getan hat, öffentlich ausgesprochen wird, wie schwere und bis jetzt noch ganz unübersehbare finanzielle Folgen die jetzt noch vielfach übliche Rentenerledigung der Dienstbeschädigung bei nervös und psychisch erkrankten Feldzugsteilnehmern haben wird. Ich stimme Herrn Hoche vollständig bei, besonders nach meinen Erfahrungen als Leiter des Beobachtungslazaretts Karlsrube und als fachärztlicher Beirat beim 14. Armeekorps. Gewiss soll jeder Beschädigte sein Recht finden, aber ein gewisser Aufbrauch, ja eine gewisse Schädigung ist bei dem Feldzug für keinen zu vermeiden, und es wäre unmöglich, alle zu entschädigen, die in irgend einer Weise später in ibrer Leistungsfähigkeit herabgesetzt sind, oder glauben, es zu sein. Bei vielen ist jetzt die Frage der Dienstbeschädigung noch nicht zu entscheiden. Ich habe deshalb eine Reihe von Patienten zeitig dienstunfähig geschrieben. Allerdings besteht auch dabei der Nachteil, dass die Frage der Dienstbeschädigung aufgerollt werden muss. Es wäre besser, wenn das einstweilen nicht geschähe. Weiterhin habe ich häufig längeren Urlaub befürwortet und ich habe nicht selten gesehen, dass während eines solchen in der gewohnten häuslichen Beschäftigung die nervösen Beschwerden zurücktraten, und dass vollständige Leistungsfäbigkeit wieder eintrat. Der Vortrag des Herrn Hoche ist um so angebrachter, als unsere Diskussionen über nervöse Erkrankungen in diesem Feldzuge etwas in das Laienpublikum, ja in das Soldatenpublikum eingedrungen sind.

Zweifellos ist die Kapitalabfindung anzustreben anstatt des Rentenempfanges. Nur glaube ich, dass, wenn, wie Herr Hoche meint, bei teilweise Erwerbsunfähigen eine 2-3fache Multiplikation der Rente als Grundlage der Kapitalabfindung genommen wird, nach kurzer Zeit schon die betreffenden Er- 
krankten in irgend einer Form nach Aufzehrung ihres erhaltenen Kapitals der Oeffentlichkeit wieder zur Last fallen werden.

Der Bemerlsung des Herrn Schultze-Bonn habe ich anzufügen, dass blaurote Verfärbung der Extremitäten doch wohl nicht nur durch das Herunterhängen bei gelähmten Gliedern entstehen kann, vielmehr auch andere Ursachen für die Verfärbung vorhanden sein müssen. Denn man sieht eine solche auch bei Nichtgelähmten, ebenso sieht man sie im Gesicht, wo ja das Moment der Zirkulationserschwerang nicht in Betracht kommt. Und eine solche Verfärbung sowohl im Gesicht als auch an den Extremitäten sieht man relativ häufig nach Verletzungen durch schwere Gewalt, besonders vom Rücken her, wie Fortschleuderung, Verschüttung. Könnte nicht dabei eine Sympathikusschädigung zugrunde liegen?

Herr Hübner-Bonn: 1. Trotz mancher Bedenken gegen die Kapitalabfindung (Fortbestehen der Invaliden-Rentenansprüche, soziales Verhalten der Zurückkehrenden) empfiehlt Hübner dieselbe doch dringend. Selbst gewisse Misserfolge, die sicher zu erwarten sind, sollten von ihrer Anwendung nicht abhalten, denn die Erfolge bei der Abfindung privater Ansprüche der Neurotiker haben einwandfrei gelehrt, dass dadurch das Leiden und namentlich die Arbeitsfreudigkeit günstig beeinflusst werden. Das Ideal würde da erreicht werden, wo Kapitalabfindung und Aufnahme der alten, oder einer neuen Beschäftigung zu erreichen ist.

2. Zur Simulationsfrage berichtet Hübner über einen Fall, in dem ein 25jähriger Mann zur Erreichung eines bestimmten Zieles eine Melancholie simulierte. Nahrungsverweigerung konnte er nur 3 Tage vollständig durchführen, nahm aber auch dann nur wenig zu sich. Auf die Suizidgedanken worde er durch ungeschickte Suggestivfragen des Arztes aufmerksam gemacht. Im übrigen glückte der mehrere Monate durchgeführte Versuch vorwiegend deshalb, weil die Sprache des Patienten schlecht verstanden wurde.

Herr Saenger-Hamburg: Saenger tritt der von Hoche vertretenen Ansicht der Kapitalabfindung auf Grund seiner Erfahrungen voll und ganz bei, von denen er einen charakteristischen Fall mitteilt.

Betreffs der Bewertung der Gesichtsfeldeinschränkung verweist er auf seine früheren Arbeiten, speziell auf seine 1896 bei Enke erschienene Schrift "Die Beurteilung der Umfallnervenerkrankungen."

Herr Neumann-Karlsruhe: Bei dem Kaufmann'schen Verfahren der nUeberrumpelungsbehandlung" psychogener Störungen handelt es sich um eine logisch wohldurchdachte und mit äusserster Konsequenz durchgeführte Behandlungsmethode einer bestimmten Art hysterischer Sy up tome, nämlich der bei Kriegsneurotikern so häufig als pathologische Reaktion auftretenden Hyper-, Dys- und Akinesien, nicht etwa um eine Behandlung der Hysterie als solcher. So aufgefasst stellt das Verfahren ein wertvolles Hilfsmittel im Rahmen der allgemeinen Möglichkeiten der Psychoneurosenbehandlung dar, das seinen Zweck rasch und zuverlässig erfüllt und auch in veralteten Fällen anwendbar ist. Besonders wertvoll ist es auch in wirtschaftlicher Hinsicht infolge seiner: oft sehr stark reduzierenden Wirkung auf die Rentensätze. 
Herr Wollenberg-Strassburg erklärt seine Zustimmung zu der von Hoche gegebenen Anregung. - Was die heute rorgestellten Fälle betreffe, so glaube er, ihren psychogenen Charakter gebührend betont zu haben. Er halte es aber für möglich, dass unter den peripheren Reizen, welche dann weiterhin von der Psyche verarbeitet würden, auch lokale vasomotorische Störungen eine Rolle spielen, die auf mechanisohem Wege zustande kämen. - Hinsichtlich der Behandlung könne er den Optimişmus des Herrn Kaufmann nicht teilen. Auch in Strassburg habe man bei gewissen Fällen gute Heilerfolge erzielt, vielfach seien aber alle Bemühungen erfolglos geblieben, obwohl für diese Fälle eine im Sinne des Herrn Ka ufmann sehr geeignete Persönlichkeit zur Verfügung gestanden habe. Es gebe eben Kranke, die für alle Fremdsuggestionen gewissermassen „gesperrt" seien und schliesslich von sich aus gesund werden, wenn gewisse unbekannte innere Motive in Wegfall kämen. W. führt ein Beispiel hierfür an.

Herr Nonne-Hamburg teilt mit, dass er nach verschiedenen Infektionskrankheiten (Typhus, akuter Gelenkrheumatismus, Erysipel, Influenza, Angina) nicht selten ausgesprochene hysterische Symptom-Komplexe habe auftreten sehen, besonders Astasie und Abasie, Monoplegie und Paraplegie, mit und ohne Sensibilitätsstörungen sowie mit und ohne trophische Störungen. Nonne entsinnt sich nicht, derartiges vor dem Kriege geseben za baben. Einen so hohen Prozentsatz von Erfolgen wie Kaufmann sie mit seinen starken elektrischen Strömen sowie seiner Suggestionsmethode hat, hat Nonne mit Hypnose nicht. Nonne beseitigt die hysterischen Symptomkomplexe in $40-60$ pCt., in ca. $60 \mathrm{pCt}$. in den früheren Monaten, beim D. U.-Material in ca. $40 \mathrm{pCt}$. Die Katamnese betreffs des Geheiltbleibens ist der Lage der Verhältnisse nach ausserordentlich schwierig. Nonne konnte bisher nur in 8 Fällen Katamnese naob längerer Zeit erheben; davon erwiesen sich 7 Fälle als geheilt geblieben. Allerdings waren die Fälle alle nur im Etappen- oder im Garnison-Dienst beschäftigt, nicht in der Front. Einer war rückfällig geworden. Von einigen Rückfällen wurde Nonne aus anderen Hamburger Lazaretten berichtet, obne dass die einzelnen Fälle ihm bisher aufgegeben worden sind. Auch Nonne hat gefunden, dass die Fälle von Stottern und ron Tachypnoe und anderen Atemstörungen besonders hartnäckig sind, sowohl gegen die Hypnose als auch, wenn die Hypnose gelingt, gegen die therapeutische Suggestion in Hypnose.

Herr Rosenfeld-Strassburg weist gegenüber den Ausführungen von Kaufmann darauf hin, dass sich in den Fällen mit Lähmung und namentlich auch in den Fällen mit Tremor und Tikbewegungen mit weniger energischen und robusten Methoden doch auch gute therapeutische Resultate erreichen lassen. Unter seinen Fällen mit Lähmung sind auch solche, die auf die zuerst angewendete robuste Therapie sogar sehr ungünstig reagierten. Arbeitstherapie und Beschäftigung im Beruf hatten in mehreren seiner Fälle einen vorzüglichen Erfolg; andere Fälle reagierten auf hypnotische Behandlung sehr günstig; eine kleine Anzahl von Fällen, darunter namentlich solche mit starken angioneurotischen Störungen, trotzte jeder Behandlung.

Herr Assmann-Leipzig schliesst sich voll und ganz Hoche an. 
Herr Lotmar-Bern: Eine Erklärung der Akrozyanose und dergl. bei funktionellen Lähmungen aus mechanischen Bedingungen ist nicht möglich; es handelt sich um echte vasomotorische Störungen, die der Lähmung koordiniert sind (di Gaspero).

Insoweit kann ich mich Herrn $\mathrm{N}$ ägeli njcht anschliessen.

\section{Sitzung am 4. Juni, vormittags 9 Uhr.}

Vorsitzender: Herr Hoche-Freiburg i. Br.

Als Versammlungsort für das nächste Jabr wird wieder Baden-Baden verabredet.

Zu Geschäftsführern werden die Herren Prof. Gaupp-Tübingen und Sanitätsrat Dr. Zacher-Baden-Baden gewählt.

16. Herr Kehr-Hamburg: "Psychologische Untersuchungen bei Kopfschussverletzten mit Bericksichtigung der Granatexplosionen."

Seit Beginn dieses Jahres habe ich im psychologischen Laboratorium in Hamburg Untersuchungen zur Beobachtungsbereitschaft von Personen durchgeführt. Die Versuchsanordnung erschien geeignet, um psychologische Aufmerlsamkeitsversuche an solchen Patienten vorzunehmen, die entweder an den Folgen einer Verschüttung oder einer Schreckwirkung von Granat- und Minenexplosionen oder auch durch Gehirnverletzungen leidend waren. Die Anregung zu diesen Untersuchungen an Verwundeten verdanke ich Herrn Professor Sänger, der mir auch in liebenswürdigster Weise die in Frage kommenden Patienten zur Untersuchung überwies.

Die Versuohsanordnung selbst ist kurz die folgende. Die Versuchsperson schaut durch den regulierbaren Spalt eines Schirmes gegen eine rotierende Trommel. Ueber diese bewegt sich ein beliebig langer Papierstreifen (in unserem Fall ca. $18 \mathrm{~m}$ lang und $17 \mathrm{~cm}$ hoch), der durch ein geeignetes Rollensystem elektromotorisch bequem auf- und abgerolit werden kann. In 3/4 Höhe des Papierstreifens ist eine Buchstabenreihe in nicht regelmässiger Reibenfolge der Buchstaben aufgedruckt. Von diesen Buchstaben erblickt die Versuchsperson, indem sie durch den Spalt schaut, immer nur einen. Derselbe ziebt verbältnismässig langsam durch das schmale Gesichtsfeld, das von dem Spalt dargestellt wird, es folgt ihm sogleich ein zweiter Buchstabe usw. Man verabredet mit der Versuchsperson bestimmte Buchstaben, in unseren Versuchen gewöhnlich drei, hei deren Auftreten im Gesichtsfeld sofort ein Zeichen zu geben ist.

Dieses Zeichen besteht im Niederdrüclien eines Tasters. Dadurch wird ein Strom gescblossen und vermittels eines elektro-magnetischen Kapillarschreibers ein Zeichen auf dem rotierenden Papierstreifen markiert. Durch die Kombination eines Zeitschreibers mit dem Tasterschreiber und durch eine bestimmte Methode der Einstellung des Apparates und der Verrechnung ist es nun möglich, die Zeitdauer jeder Reaktion in 25 stel Sekunden genau abzulesen. Es entstehen 
dadurch Tabellen von Reaktionszeitwerten, bei unseren Versuchen ca. 150 Wertefür 150 aufeinanderfolgendo Reaktionen. Diese verteilen sich über die Gesamt. dauer des Versuchs, ca. 13 Minuten. Es wird nun das Mittel der Reaktionsdauer für je $1 / 2$ Minute berechnet, und diese Mittelwerte werden in einer Kurve. graphisch dargestellt. Ferner wird die Schwankung der Reaktionszeit von Versuch zu Versuch tabellarisch anfgestellt, auch davon das Mittel für jede halbe. Minute berechnet und gleichfalls in einer Kurve bildlich dargestellt.

Man erhält auf diese Weise für jeden Versuch zwei Kurven mit zwei Gesamtmittelwerten. Der Verlauf dieser Kurven ist höchst charakteristisch. Zu Grunde gelegt werden die Kurven von Gesunden, und zwar von solchen, die im pralstischen Leben, d. h. in ihrem Beruf eine zuverlässige und sichere. Aufmerksamkeitsleistung ausüben. Während nun bei diesen Versuchspersonen der Versuch noch ein reiner Beobacbtungsversuch bleibt, bei dem die Reaktionsdauer zu Anfang und zu Ende ziemlich gleich ist, wird er bei manchem Verwundeten schon zum ausgesprochensten Ermüdungsversuch. Dies erkennt man an dem aufälligen Anstieg der Reaktionszoitkurven. Damit verknüpft ist sowohl eine grosse mittlere Schwankungsbreite, innerhalb deren die Reaktionszeiten sich bewegen, als auch eine grosse Unregelmässigkeit dieser Schwankang von Versuch zu Versuch. Die Anzahl der Versuche, die bis jetzt durehgeführt werden konnte, ist noch zu gering, um bereits eine allgemeine Klassifikation zu gestatten, doch sei nur soviel gesagt, dass die Kurve genannter Art am. charakteristischsten sich ergeben hat bei einem Psychopathen, der durch die Folgen einer Verschüttung aus dem Felde zurückkam. Einen ähnlichen Verlauf ergaben bisher Fälle der Schreckwirkung. Gehirnverletzungen, soweit solche Fälle bisher untersucht wurden, lassen dagegen viel weniger Ermüdungserscheinungen im Verlauf der Kurve erkennen. Wobl aber eine grosse und unregelmässige Schwankung in der gegenseitigen Abweichung der Realktionsdauer von Versuch zu Versuch.

Obwohl wir noch am Anfang dieser Versuche stehen, so erscheint die. Methode doch geeignet, neben die klinischen Methoden zu treten, besonders um über den psychogenen oder organischen Charakter des Zustandes ein psychologisch-objektives Urteil zu erstreben and dies wioderum verwertbar zu machen für die Zwecke der Berufsberatung, der Rentenabfindung und anderer, dureh die psychologische Eigenart der Verwundeten stark bedingter Entscheidungen. Ein Zusammenarbeiten des Neurologen und Psychologen ist dabei natürlich erste Voraussotzung.

(Eigenbericht.)

17. Herr Gerhardt-Würzburg: „Ueber funktionelle Störungen. bei organischen Nervenkrankheiten."

Die Lokalisation und Ausdehnung vieler zentraler und auch wancher peripherer Lähmungen wird nicht nur durch die anatomischen Verhältnisse am Ort der Erkrankung, sondern ancb durch funktionelle Momente bedingt.

Für die motorischen Lähmungen kommen hier neben den bekannten Unterschieden im Verhalten der Strecker und Beuger in betracht teils Ueberanstrengung und Abnutzung einzelner Muskeln oder Muskelgruppen (Edinger's. 
Aufbrauchtheorie), teils funktionelle Zusammengehörigkeit scheinbar getrennt entspringender Muskeln Serratus und Trapezius bei zentraler, aber auch bei peripherer Lähmung, Schultergürtelmuskeln bei spinaler Muskelatrophie, teils geringere Widerstandsfähigkeit einzelner Nerventeile (Posticuszwcig des Laryng. inf., Poroneus gegenüber dem Tibialis).

Noch komplizierter liegen die Verhältnisse bei sensiblen Lähmungen. Rindenberde können Anästhesie an eng umschriebener Stelle (Daumen, Mundwinkel), aber auch Anästhesie in längsstreifenförmiger Begrenzung, die an segmentäre Verbreitung erinnert zur Folge haben; ferner kommen bei zerebralen und diffusen spinalen Erkrankungen an Rumpf und Oberarmen Hypästhesien im Bereich der Segmente C 8 bis D 4 vor, die sich andeutungsweise schon bei manchem Gesunden finden und deshalb mit Wahrscheinlichkeit auf eine von Haus aus geringere Widerstandsfähigkeit dieser Gebiete zu beziehen sind. Am Bein besteht eine derartige Disposition im Bernhard'schen Feld (N. cut. fem. ext.), die öfters bei Tabes, aber auch bei spinaler Herderkrankung (Wirbelschüsse) deutlich wird.

Dazu kommt noch die allgemeine Prädisposition der distalen Teile für sensible Störungen. Diese für zerebrale Lähmungen bekannte Eigentümlichkeit lässt sich auch bei vielen diffusen und herdförmigen Spinalleiden auffinden, so auch bei der Rückbildung von Paraplegien nach Wirbelschüssen. Diese Disposition der distalen Teile für die Anästhesie kann praktiscbe Bedeutung bekommen, wenn sie einen zu tiefen Sitz des Herdes vortäuscht, ferner bei Wirbelverletznngen, wo das Herabrücken der Anästhesiegrenze das erste Symptom sein kann, dass keine totale Querschnittstrennung vorliegt, endlich bei der Differentialdiagnose zwischen organischer und funktioneller, namentlich funktionell überlagernder Anästhesie.

(Eigenbericht.)

18. Herr C. von Hess-München: „Vorfübrung eines Apparates zur messenden Bestimmung von Stärungen des Pupillenspieles" (Differential-Pupilloskop).

Hess betont die Notwendigkeit, Störungen des Pupillenspieles schon wor dem Eintreten der reflektorischen Starre zu erkennen und den Grad solcher Störungen messend zu bestimmen; er hat zur Lösung der Aufgabe einen bisher noch nicht begangenen Weg eingeschlagen, indem er die pupillomotorische Unterschiedsempfindlichkeit ermittelt, d. b. die kleinsten Lichtstärkeunterschiede, die in gesunden und kranken Augen eben merkliches Pupillenspiel auszulösen vermögen. Er führt den Apparat vor, der solche Messungen in einfacher Weise mit überraschender Genauigkeit auszaführen gestattet, und mit dessen Hilfe er unter anderem auch das Problem der objektiven Untersuchung des K'arbensinnes zu lösen vermochte. Er weist insbesondere auch darauf hin, dass es mit dem Apparat möglich sein wird, die der reflektorisohen Starre bei Tabes vorausgebenden Krankheitsstadien, über die bisher so gut wie alle Kenntnisse fehlen, zu untersuchen und so die Erkrankung wesentlich früher zu erkennen, als es bisher möglich war.

(Eigenbericht.) 
19. Herr Bayerthal-Worms: "Zur Lehre ron den psychischen Funktionen des Stirnhirns."

Nachdem es sich für das schulpfliehtige Alter als eine gesetzmässige Erscheinung herausgestellt hatte, dass mit abnehmender Kopfgrösse gute intellektuelle Fähigkeiten seltener, minderwertige dagegen immer häufiger vorkommen, so dass schliesslich an dem einen Ende der Reibo bei den kloinsten Köpfen nur intellektuelle Minderwertigkeit zu finden ist, suchte Vortragender zu ermitteln, welche Rolle dabei die Grösse des Vorderhauptes spielt. Er fand hier die gleiche Gesetzmässigkeit, während ein Zusammenhang der Intelligenz mit der Grösse des Hinterbauptes nieht nachweisbar war. Vortragender erblickt darin einen von manchen Autoren noch vermissten Beweis dafür, dass dem Stimbirn eine besonders wichtige Rolle tür die intellektnellen Verrichtungen zukommat. Vielleicht kann man das vorliegende Problem nach der quantitativen Seite am klarsten formulieren, wenn man unter Berücksichtigung der Altersund Geschlechtsunterschiede a. a.m. fragt, wie gross das Stirnhirn baw. der Stirnanteil des. Kopfes mindestens sein muss, wenn nicht von rornherein gute, d. b. wesentlich über dem Durchschnitt stehende intellektuelle Fähigkeiten ausgeschlossen sein sollen. Voraussichtlich wird es mittels plastischer Nachbildungen des Kopfes, die eine Volumenbestimmung gestatten, gelingen, der Lösung der Frage näher zu kommen.

(Ausfuhrliche Veröffentlichung wird an anderer Stelle erfolgen.)

(Eigenbericht.)

20. Herr Aschaffenburg-Cöln: „Ueber die Spätfolgen der Sohädelverletzungen."

Die Erfahrungen des Ietzten Jahres haben über viele Pankte, die auf dex Versammlung im vorigen Jahre noeh strittig sein konnten, Licht verbreitet und insbesondere orkennen lassen, dass die Gefabren der Streif- und Tangentialschüsse bei weitem unterschätzt worden sind. Die lebendige Kraft, mit der ein Geschoss den Schädel trifft, ist bei Nahschüssen so gross, dass das ganze Gehirn mit grosser Wucht in Bewegung gesetzt wird und seinerseits eine erhebliche Druckwirkung ausüben kann. Jedes eindringende Geschoss verdrängt ausserdem einen Teil des Gehirns und seine Wirkung wird um die verdrängten Gehirnmengen vermebrt, die im Ausweichen auf die fernere Umgebung starken Druck ausüben. Bei Tangentialschüssen kann es ohne Verletzung des Knochens zu Absplitterungen der T'abula vitrea kommen, deren Splitter radiär ins Gehirn geschleudert werden.

Alle diese mechanischen Vorgänge machen verständlich, weshalb wir neben der unmittelbar von dem Schuss getroffenen Gehirngegend auch Störungen an weiter abgelegenen Stellen finden, und weshalb sich im Anschluss an scheinbar nicbt sehr erbebliche Zertrümmerungen von Hirnsubstanz eine allgemeine Demenz entwickeln kann, die doch nur bei der Annahme weitverbreiteter Hirnveränderungen verständlich ist. Aehnlich, wie Scbädelschüsse, kann grobe Gewalt, die den Schädel trifft, z. B. bei Anschlagen des Kopfes bei Verschüttungen, wirken. 
Unter solchen Umständen werden wir neben den lokalisierten Ausfallserscheinungen mehr oder weniger verbreitete Allgemeinstörangen und Spätfolgen el'warten müssen. Unter den Allgemeinstörungen sind, abgesehen von den subjektiven Beschwerden, besonders zu nennen: Schnelle Ermüdbarkeit, allgemeine Erschwerung des Auffassens und Dønkens, eine erhebliche Störung der Merkfähigkeit (boi verhältnismässig wenig gestörtem Gedächtnis), eine Neigung zu Kongestionen und Schwindelzuständen, Alkoholintoleranz, Abstumpfung des Gefühlslebens bei gleichzeitiger gesteigerter Erregbarkeit.

Neben diesen Allgemeinstörungen bestehen zwei Gefahren: die Entwicklung der traumatischen Neurose und der Epilepsie. Die traumatische Neurose ist bei wirklich ernsten Gehirnschädigungen nicht allza häufig; vielleicht weil die Stumpfheit eine Art Gegenmittel gegen das Sichvertiefen in die Beschwerden und Folgen darstellt.

Um so bedenklicher ist das Auftreten epileptischer Anfälle, die sich nicht etwa nur bald nach der Verletzung infolge unmittelbare: Hinneizung durch Knochensplitter oder - verletzungen zeigen. Vortragender berichtet über einen Fall, in dem sich 11 Monate nach einem schnell geheilten Hirnschuss, nachdem der Verletzte sich schon lange verhältnissmässig wohlgefühlt hatte, nach den ersten drei volldurchgearbeiteten Tagen ein Status epilepticus entwickelte; ohne Druckstejgerung, wie sich bei einer Lumbalpunktion ergab; die beiden Trepanöffnungen an der Stirn hätten auch genügt, jedes übermässige Anwachsen des Drucks im Gehirn zu verhindern. Trotz aller Bemühungen starb der Kranke nach wenigen Stunden. Die Autopsie ergab eine Verwachsung beider Hirnpole mit der Dura und der Dura mit der darüberliegenden Haut in ganz mässigem Umfange, starke Blutüberfüllung der Hirnrinde, abor keinen Anhaltspunkt für entzündliche Vorgänge.

Hier hatte also eine kurze Arbeit genügt, um eine todbringende Epilepsie hervorzurufen. Auch in zahlreichen anderen Fällen traten die ersten Erscheinungen der Epilepsie nach kurzdauernder körperlicher oder geistiger Arbeit auf. Von besonderer Bedeutung ist auch die Tatsache, dass sich epileptische Anfälle. nach Schädelverletzungen nicht nur da finden, wo die motorischen Zentren durch Fremdkörper oder entzündliche Prozesse gereizt werden, sondern dass die Neigung des Gehirns zu Bewusstseinstrübungen mit und ohne Krämpfe auch da zu beobachten ist, wo kein unmittelbarer lokaler Reiz zu finden ist. Solche Erfahrungen zwingen, äusserst vorsichtig bei Uebungsversuchen und bei der Rückkehr zur Arbeit zu sein; vielleicht wird das geschädigte Gewebe schneller "verbraucht" wie das gesunde. Wir können nicht wissen, wie weit die Widerstandsfähigkeit des Gehirns geht.

Jedenfalls wird man in keinem Falle berechtigt sein, den Kopfschussverletzten die Strapazen des Feldzugs und anstrengenden Garnisondienstes zuzumuten.

Durch die Gefahr, dass sich noch lange Zeit nach völliger chirurgischer Heilung Epilepsie herausbilden kann, wird die soziale Brauchbarkeit der Kopfverletzten, die ohnedies durch die Allgemeinschädigungen schwer geschädigt sind, noch erheblich rerringert, und es ist nicht zu leugnen, dass die Zukunfts- 
aussicbten für alle Fälle von Schädelverletzungen mit Ausnahme solcher durch kraftlos aufprallende Geschosse durchweg überaus traurig sind.

(Rigenbericht).

21. Herr Weygandt-Hamburg: "Ueber Degeneratio adiposogenitalis."

So klar allmählich sich auch die Pathogenese der Akromegalie als Ueberproduktion des vorderen Hypophysenteils infolge Vermehrung der eosinophilen Zellon heraushebt und auch das Bild der Adiposogenitaldystrophie auf Funktionshemmung der Neurohypophyse zu beruhen scheint, ja selbst der hypophysäre Zwergwuchs allmählich mebr Boden gowinnt, so sind doch die offenen Probleme des grossen Gebiets noch mannigfach und schwierig genug, die atypischen Fälle sind recht zahlreich, und die Bedentung sekundärer Hypophysensymptome bei sonstigen Hirnerkrankungen wird noch keineswegs immer hinreichend gewürdigt. Selbst die Akromegalie, deren Lehre am festesten gefügt erscheint, gibt noch reichlich Rätsel auf: So wurden Fälle beobachtet von Vermehrung der Eosinophilen im vorderen Hypophysenteil ohne Akromegalie, ferner von Adenoepitheliom bei Akromegalie mit vorwiegenden chromophoben Zellen. Als atypische Vorkommnisse bei Akromegalie sind zu erwähnen die Leontiasis, dann die Vergrösserung der Zäbne, ferner die Verlängerung der Phalangen, Metakarpen, Metatarsen. Bemerkenswert ist, wie sich die akromegalen Schwangerschaftssymptome manchmal selbst bei Pluriparen sogar im 4. Jahrzehnt noch in einem vermehrten Längenwachstum äussern. Besonders schwierig zu beurteilen sind die Beziehungen der Akromegalie zur Idiotie, aber auch zu funktionellen Psychosen. wie den manischen und depressiven Prozessen.

Mag auch das typische Bild der Adiposogenitaldystrophie gelänfig sein, so möchte ich doch auf einige atypische Formen eingehen. Man findet ja primäre Erkrankung der Neurohypophyse durch Anlagemangel, durch Tumor, durch Infektion wie Tuberkulose und Lues, und durch Kachexie. Dann aber auch sekundäre Störung, vorwiegend durch anderweitig lokalisierten Tumor, ferner durch Meningitis serosa.

Zwei der folgenden Fälle habe ich früher schon zitiert, gegenwärtig aber liegt ihr Obduktionsbefund vor.

Ein geistig schwach beanlagtes Mädchen, das schon lange am linken Bein paretisch war, bekam Menses mit 15 Jahren, die wieder aufhörten mit 161/2 Jahren. Gleichzeitig traten Kopfweh, Schwindel, Bewasstlosigkeit, Hinfallen, unwillkürliche Bewegungen der Finger und Gesichtsmuskeln links, Sehscbwäche, Urininkontinenz, Teilnahmlosigkeit und Schlafsucht auf. Die Pupillen waren different und lichtstarr, die Staungspapille ging bald in Sehnervatrophie mit Amaurose äber, die Sehnenreflexe waren gesteigert, links mehr als rechts. Der Spinaldruck war erhöht, der 'Jürkensattel im Röntgenbilde war erweitert mit Schwund der hinteren Fortsätze. Die Untersuchungen auf Wassermann, Globalin, Pleozytose verliefen negativ. Zeitweise war Sondenernährung nötig. Es stellten sich epileptiforme Anfälle ein. Vorübergehend erfolgte psychische Besserung. Mit $171 / 2$ Jahcen nahm das Gewicht rasch 20 , von $35 \mathrm{~kg}$ 
auf 70, es bildeten sich Hängebauch, Hängebrüste. Bald darauf kehrten die Menses wieder zurück. Der Zustand war fermerhin recht wechselnd, Erbrechen, Mattigkeit, Somnolenz, tonische Krämpfe mit Bewusstlosigkeit folgten auf Zeiten einer läppisch heiteren Stimmung mit Neigung zu plaudern, zu singen und za seherzen. Fine Seitenventrikelpunktion brachte subjektiv etwas Besserung. Die Haut wies an Brust und Bauch etwas Pigment auf. Mit 20 Jahren wog Pat. $79 \mathrm{lrg}$.

Das Blutserum baute nach Abderhalden Hypophyse ab. Es wurde Pituglandol gegeben, das Gewicht ging langsam berab, zeitweilig fühlte sich Patientin subjektiv ganz wohl, war freundlich und zufrieden, auch wieder zeitlich und örtlich orientiert, rechnete anch leichte Aufgaben. Polyurie ging bis $2800 \mathrm{ccm}$, es war alimentäre Glykosurie nachweisbar. Die Genital- und Achselhaare waren sehr spärlich. Später traten Blutungen am Zahnfleisch und an den Beinen auf. Der Zustand wurde immer kachektischer, das Gewicht sank auf $33 \mathrm{~kg}$, die Menses zessierten wieder. Unter Kollaps erfolgte der Tod.

Der Gesichtsschädel zeigte Prognathio, so hochgradig wie bei einem Senegalneger.

Die Schädelknochen waren ausserordentlich dünn und stellenweise usuriert. Das Hirn war ödematös, die Windungen platt. An der Basis nach dem rechten Thalamus zu fand sich eine fast hühnereigrosse, harte Geschwulst, doch schob sich ein fast taubeneigrosses, zystisches Gebilde dazwischen. Die Tumormasse erstreckte sich diffus in die Brückensubstanz binein, doch zeigte sich hier bis zum 4. Ventrikel zu wieder ein zystisches Gebilde. Die Ventrikel sind sehr weit. Die Hirngefässe sind zart. Das Hirn wog 1320 g. Die Hypophyse $(0,55)$ war knopfartig plattgedrückt.

Histologisch charakterisierte sich der Tumor als ein Gliosarkom. Die Hypophyse zeigte ganz überwiegend nur noch normales Drüsengewebe und lediglich als kleinen Randstreifen noch Neurohypophysengewebe, das an sich nicht krankhaft verändert erschien.

Die Hirnrinde zeigte enge, aber regelmässige Zellanordnung, keine Gliavermehrung, keine Gefässvermehrung, keine Merkmale einer Entzündung. Die kleineren Pyramiden erinnern etwas an rundliche, embryonale Zellen, in der Schicht der grossen Pyramiden finden sich Zellen mit dicht geschwollenem, ungefärbtem Kern und mit Zellprotoplasma von wabiger Struktur. Thyreoidea, Nebenniere und Ovarium waren normal.

Bemerkenswert in diesem Falle ist, dass der Erkrankung, die in frühe Jugend zurückwies, ein Tumor der Hirnbasis zugrunde lag, und dass trotz schwerer Kompression der Neurohypophyse die genitale Komponente wenig ausgeprägt war; wohl trat früh Menopause auf, aber mit Beginn der Adiposität kan die Periode wieder.

Bei dem geschilderten Fall trat die hochgradige Fettsucht im Laufe der Kacbexie zurück, und es kam zu extremer Abmagerung. In einem anderen Fall fand ich eine partielle Lokalisation der Fettsucht, in der unteren Körperhälfte, mit vorspringdem Bauch, breitem Becken und dicken Fettpolstern der Unterund besonders der Oberschenkel, bei schmalem Schultergürtel. 
Soweit ich bei unseren Fällen eine Untersuchung des Serums oder Urins nach $\mathrm{Abderhalden}$ durch $\mathrm{K}$ afka vornehmen lassen konnte, ergab sich meist, aber nicht immer ein Abbau der Hypophyse. Wenn dieser in einzelnen kilinisch ganz ausgeprägten Fällen, die in Verbindung mit Schwachsinn bis in die frübeste Jugend zurückreichen, nicht nachweisbar war, so ist dies eben aus der Afunktion des Organs im Gegensatz zu der noch Abbau aufweisenden Dysfunktion zu erklären. Abbauunterschiede allerdings gegenüber dem hypophysären Längenwuchs und der Akromegalie lassen sich noch nicht feststellen. Im ganzen findet sich bei akromegalen Wachstumsveränderungen Hypophysenabbau häufiger als bei Adiposogenitaldystrophie. Ich bin überzengt, dass die mannigfachen Gegner der Abderhaldendialyse am ehesten durch deren Ergebnisse bei innersekretorischen Störungen zur Erkenntnis von der Bedeutung des Verfahrens bekehrt werden können. Neben bypophysären kommen die dystbyreoiden Erkrankungen, aber auch noch manche andere in betracht. Geradezu frappant waren zwei Fälle, die ich neuerdings untersuchen liess: Ein 13jähriger Knabe mit psychischer, doch nicht somatischer Pubertas praecox, zeigte lediglich Nebennierenabbau; und der kürzlich von $O b \operatorname{man} n$ beschriebene böchst interessante Meininger Fall von hochgradiger Pubertas praecox ergab bei der Abderhalden-Dialyse in unserm Laboratorium Abban von Hoden, Thymus, Nebenniere und, soweit das Präparat verwertbar war, von Epiphysis cerebri.

Viel zu wenig beachtet wird der hypophysäre Schwachsinn, der, abgesehen von zerebralen Reizerscheinungen bei Tumor, gewöhnlich ein ganz eigenartiges psychisches Bild darbietet, indem es sich um eine Demenz verschiedenen Grades, aber von ausgesprochener erethisch-euphorischer Eigenart handelt. Die läppische Heiterlkeit und aufdringliche Unruhe steht dabei in einem grotesken Gegensatz zu der durch die Fettsucht bedingten Unbebolfenheit.

In einem Falle, dessen Hirn ich Geheimrat Sander verdanke, waren mit $1^{1} / 2$ Jahren epileptoide Krämpfe und darauf schwere Diphtherie aufgetreten, wodurch er die Anfänge des Sprechens wieder verlernte. Er blieb geistig zurück und wurde schon früh auffallend dick. Mit 5 Jahren überstand er Typhus, mit 19 Jahren erlitt er einen Krampfanfall. In seinem Wesen war er immer wild und zerstörungssüchtig. Er konnte kaum seinen Namen sprechen und war nicht einmal mit Haarzupfen zu beschäftigen; alles liess er unter sich; er musste angekleidet werden. Vielfach spielte er mit seinen Genitalien.

Trotz Thyreoidinbehandlung stieg sein Gewicht mit 30 Jahren auf $151,5 \mathrm{~kg}$ bei $167 \mathrm{~cm}$ Körperlänge. Allmählich ging das Gewicht wieder auf $122 \mathrm{~kg}$ herab. Mit 35 Jahren starb er.

Das Gehirn (in Formol) wog nur $1055 \mathrm{~g}$, die Hypophyse $0,8 \mathrm{~g}$. Die Neurohypophyse fiel auf durch faserreiches Gewebo mit wenig Kernen. Der Drüsenteil war gut ausgebildet, ebenso die Pars intermedia.

Die Hirnrinde war schmal, besonders am Stirnpol. Die Gefässe erschienen zahlreich, doch waren keine entzündlichen Veränderungen vorhanden, ebensowenig Vermebrung der Glia und Trabantzellen. Die Schicht der kleinen Pyramiden war noch gut abgesondert, während die Anordnung der mittleren und 
grossen Pyramidenschicht und der Körnerschicht etwas verzerrt war; die Kerno waren geschwollen, wenig gefärbt, das Protoplasma zeigte eine wabig-körnelige Struktur.

Die Schilddrüse hatte ausgesprochen hypoplastischen, infantilen Bau, streckenweise mit sehr wenig oder gar keinem Kolloid. Die Testikel waren durchaus normal und liessen Spermatogenese erkennen.

Fs ist wohl eine Entwicklungshemmung auf gruad von Encephalitis in frühester Kinảheit anzunehmen.

In den beiden Fällen hat die Fettsucht das Bild beherrscht, während die Genitalstörung im ersten gering vertreten war, im zweiten eigentlich ganz fehite. Strong genommen muss man auch hier von einem sekundären Hypopituitarismus sprechen, indem die Neurohypophyse einmal durch eine entzündliche Hirnerkrankung in ihrer Entwicklung verhindert, das andere Mal durch Tumordruck in ihrer Funktion gestört wurde. Dem gegenüber wären primäre Fälle von Hypopituitarismus diejenigen, in denen der Sitz der Erkrankung, Tumor oder Tuberkulose oder Syphilis, in dem Organ selbst zu suchen ist.

Aber nicht selten trifft man auch den Fall, dass das Krankheitsbild durch anderweitige, augenfälligere Symptome beherrscht wird, und nur beiläufig, ganz sekundär, infolge Druckwirkung des Hydrocephalus internus vom Infundibulum her die Hypophysenstörung mit dadurch bedingter Fettsucht und Genitalhemmung in Erscheinung tritt.

Solche Fälle, zu denen der an zweiter Stelle geschilderte in Beziehung steht, liegen vor bei manchen Formen der Epilepsie und Porenzephalie; manchmal zeigt sich ausgesprochen femininer Habitus mit Fettsucht bei einem Epileptiker.

Weiterhin gehören die ätiologisch damit wieder verwandten Fälle von Hydrozephalie hierher, in denen wenigstens Zworgwuchs oder Minderwuchs nicht selten sind, und dio Selktion mehrfach eine ganz erweiterte Hypophysengrube ergibt. Auch bei manchen Formen yon Mikrozephalie ist Minderwuchs und Zwergwuchs vorhanden.

Besonders wichtig sind auch die Fälle von Erbsyphilis, die manchmal eunuchoiden Längenwuchs, gelegentlich aber auch Adiposität aufweisen. Ein weiblicher Fall war seit Geburt schwachsinnig, haite 6 Zehen an jedem Fuss, es bestand luische Chorioretinitis; mehrfach traten bysterische Symptome hervor: mit 30 Jahren betrug die Körperlänge $154 \mathrm{~cm}$, das Gewicht des stark adiposen Körpers betrug $87,5 \mathrm{~kg}$.

Auch bei den vereinzelt vorkommenden Fällen, dass ein Paralytiker trotz fortschreitender Kachexie allmählioh immer adiposer wird, ist an eine Beteiligung der Hypophyse zu dezken.

Ein höchst eigenartiger Fall, den ich durch v. Blomberg kennen lernte, zeigte sekundäre Adiposiät bei hochgradigem Hydrozephalus mit Chondrodystrophie.

Das Kind kam mit einer Missbildung des Schädels auf die Welt, war links blind, lernte aber mit einem Jahr laufen und sprechen. Mit 91/4 Jabren wurde sie der Anstalt Kosten überwiesen, sprach damals deutsch und polnisch, hatte gutes Gedächtnis, sang ziemlich gut, beschäftigte sich, wenn sie auch mit den 
Händen unbeholfen war. Mit 14 Jahren stand die Entwicklung still, und es kam zu Rückschritten, sie wurde unbeholfen, zeigte Inkontinenz, wurde körperlich und auch geistig hinfälliger, doch war sie zeitlebens im ganzen in heiterer Stimmungslage.

Mit zwei Jahren war eine Hirnpunktion gemacht worden. Zeitweilig traten, anscheinend seit dem Eingriff, epileptische Krämpfe auf, bis zu 26 an einem Tage, vor denen die Stirnhant eingesunken schien. Im letzten Jahr war zweimal die Menstruation spurweise vorbanden, Scham- und Achselhaare waren angedeutet. Mit $161 / 4$ Jahren starb sie im Anschluss an einen epileptischen Anfall.

Am auffallendsten war der Schädelbau, der eine Art Zylinderform aufwies, vorwiegend infolge der nach oben seitlich aufgetriebenen Stirnhöoker, zwischen ihnen die offene grosse Fontanelle, ferner durch die ganz eigenartig seitwärts getriebenen Schläfenschuppen, unter denen die Ohren scbräg nach unten verdrängt waren. Der horizontale Schädelumfang in der Höhe der Stirnhöcker betrug 42, in der Gegend der Schläfenschuppen $58 \mathrm{~cm}$, die Ohrscheitellinie betrug $56 \mathrm{~cm}$ gegen 34 in der Norm. Die Körperlänge betrug $123 \mathrm{~cm}$, das Gewicht $50 \mathrm{~kg}$.

Ganz auffallend war diø Adiposität, die bereits im Gesicht angedeutet war, dicke, schon etwas bängende Mammae, bedingte, einen Hängebauch und ausserordentlich fettreiche Ober- und Unterschenkel betraf. Auch am Herzen und den Nieren fanden sich starke Fettablagerungen.

Ferner bestand Dreizackhand mit starker Verkürzung und Verdickung der Daumenphalangen.

An den Füssen fanden sich Schwimmhäute zwischen 1. und 2., sowie 3. and 4. Zehe. Links war der erste Metatarsus doppelt angelegt.

Die Thymus wog $50 \mathrm{~g}$.

Das Grosshirn war wie eine Blase aufgetrieben; es wurden $1150 \mathrm{ccm}$ Hirnwasser aufgefangen.

Die Sichel und das Zelt waren mehrfach durchbrochen. Zwischen Schläfenlappen und Parietallappen schob sich eine $3 \mathrm{~cm}$ breite Knochenspange, aus spongiösen Knochenlamellen bestehend, ein. Das entleerte Hirn wog $1250 \mathrm{~g}$.

Das unserem Laboratorium freundlichst zur Verfïgung gestellte Hirn war in kollabiertem Zustande schwer zu untersuchen. Am auffallendsten war eine jenen zwei Knochenspangen entsprechende seitliche Einlerbung, sowie die ausserordentlicho Auftreibung infolge der Ventrikelerweiternng.

Soweit jedoch überhaupt Rinde vorhanden war, zeigte sie meist eine recht gute Zellarchitektur, besonders parietal und occipital. Nirgend fanden sich Gliavermehrang oder Spuren von Entzündung, die Gefässe waren zartwandig. Auch das Ammonshorn war gut erhalten.

In der Temporalgegend waren Zellausfall und Gliawncherung zu bemerken; die kleinen Pyramiden hatten einen etwas rundlichen Typus; die Pyramiden wiesen auch zablreiche Trabantzellen auf, ferner sah man in der Pyramidenschicht spindelförmige Zellen mit Trabantenzellen in einem anderen Winkel einstrahlen. Eingehendere Untersuchung steht noch bevor. Der ganze 
Fall ist am ehesten deutbar als eine hochgradige, auf Anlagestörung beruhende Chondrodystrophie mit Wasserkopf, wofür nicht nur der Extremitätenbau, sondern auch sogar die psychische Eigenart heranzuziehen ist. Was aber im heutigen Zusammenhang besonders interessiert, dass ist die aufallende Fettsucht. Wenn auch die Hypophyse leider nicht mit erhalten ist, lässt sich doch aus dem Bilde der Schädelbasis noch entnehmen, dass der Türkensattel platt erweitert und somit die Hypophyso einem hohen Druck ausgesetzt war. Meines Erachtens handelt es sich also bei diesem höchst eigenartigen Falle gleichzeitig auch um eine sekundäre Hypophysenfettsucbt.

Erfrealicherweise lässt sich wenigstens vereinzelt bei adiposogenitalen Störungen doch durch Darreichung von Hypophysensubstanz eine Besserung der körperlichen und psychischen Symptome herbeiführen.

Unsere Uebersicht zeigt, wie ausserordentlich mannigfach und atypisch bei den verschiedensten Hirnerkrankungen Störungen im Sinne des Hypopituitarismus das klinische Bild beherrschen oder auch sekundär zu den übrigen, auffälligen Symptomen hinzutreten können.

(Demonstration von Schädel, lebensgrossen Photographien, Röntgenbildern, Mikrophotographien der erwähnten Fälle). (Eigenbericht.)

22. Herr M. Meyor-Köppern i. Taunus: "Zur Frage der AdrenalinUnempfindlichkeit bei Dementia praecox."

Es wurde bisher bei 10 Normalversucbspersonen und 52 Fällen von Psychose die Wirkung subkutaner Adrenalininjektion $1 \mathrm{ccm}(1: 1000)$ auf Pulsfrequenz, Blutdruck und Amplitude, sowie auf Veränderungen in derZusammensetzung des Blutbildes studiert. Ueber die Hälfte der Fälle von Dementia praocox zeigten keine Veränderung oder Verlangsamung der Pulszabl, etwa ebensoviele keine Veränderung des Blutdrucks oder ausgesprochene Blutdrucksenkung. Im gieichen Sinn verhielt sich indessen auch die Mebrzahl der Fälle von angeborenem Schwachsinn, die mit einer Senkung antwortete. Die Veränderungen des Blutbildes auf Adrenalininjektion zeigten bereits bei Normalversuchspersonen derart erbebliche Schwankungen, sowohl binsichtlich der Zabl der eosinophilen, wie der Verhältniszahlen der polymorphkernigen neutrophilen zu den lymphozytären und mononukleären Formen, dass unter pathologischen Bedingungen keinerlei diagnostische Schlüsse daraus gezogen werden können.

(Eigenbericht.)

23. Herr Goldstein-Frankfurt a/M.: "Ueber kortikale Sensibilitätsstörungen."

G. hat seine Untersuchungen an Kopfschussverletzungen ausgeführt, bei denen wesentlich Rindenverletzungen vorlagen. Er beschränkt sich auf die Besprechung der Ausbreitung der sensiblen Störungen in der Peripherie und behält sich vor, über die Störungen der einzelnen Qualitäten an anderer Stelle zu berichten.

In der Literatur werden über die Ausbreitung der sensiblen Ausfälle bei kortikalen Herden zwei Anschauungen vertreten. Nach der einen, der älteren, 
sind die distalen Abschnitte des Körpers stärker als die proximalen betroffen; und die Grenzlinien zwischen gestörten und ungestörten Gebieten verlaufen. etwa ringformig um den Körper resp. die Glieder. Nach der zwoiten lommen auch bei kortikalen Läsionen Sensibilitätsstörungen von segmentalem Typus vor. Die Existenzberechtigung der letzteren Annahme wird lebhaft bestritten. Es wird gesagt, dass die letzteren Störungen, die sich besonders auf die Hand beziehen, nur scheiabar segmentaler Natur sind, dass es sich dabei vielmehr um eine Schädigung von Zentren für ganz bestimmte Empfindungsmechanismen handelt, die ähnlich wie die einzelnen Bewegungsmechanismen in der Rinde vertreten sind, und deshalb isoliert geschädigt werden können. Mit den Segmentsausbreitungen haben diese Anfällo nichts zu tun. In letzter Zeit hat besonders Marburg diese Anschaung vertreten und auf den besonderen Mechanismus, den die zum Tasten besonders wichtigen 1., 2. und 3. Finger darstellen, hingewiesen.

Nach seinen eigenen Untersuchungen unterscheidet Goldstein $3 \mathrm{Haupt}$ typen von Störungen, die er der Versammlung an zahlreichen Bildern illustriert.

I. Typus: Diffuses Betroffensein der ganzen Körperbälfte mit besonderer Beeinträchtigung der Extremitäten. Bei der Restitution bleibt die Störung am schwersten in der Peripherie der Extremitäten und der Seitenpartio des Rumpfes und Kopfes, An den Extremitäten schneidet sie ringförmig, entweder an Hand-, resp. Fussgelenk ab oder in verschiedenen Abschnitten der. Finger. Dorsalfläche und Volarfläche sind nicht immer gleich betroffen.

Als Erklärung bierfür ist anzunehmen, dass das Feld der bewussten Sensibilität um so umschränkter — wenn auch feiner organisiert _- ist, je peripherer der Körperabschnitt liegt, für den Rumpf also grösser als für die Hand. Nur so orklärt sich, dass 1. zwar die Hand isoliert geschädigt jst, aber nie der Rumpf, 2. dass die Hand immer schwerer geschädigt ist als der Rumpf.

II. Typus: Ausschliessliches Befallensein der medialen oder lateralen Hälfte der Hand oder des Fusses bei evtl. mehr oder weniger vollständigem Freisein des übrigen Körpers. Selten betrifft die mediale Störung nur den 4 . und 5. Finger nebst zugehörigem Handteil und. die laterale den 1., 2. und 3. Finger; meist ist auch die andere Hälfte mehr oder weniger mitbetroffen. Nicht selten ist nur der Daumen resp. der kleine Finger isoliert betroffen oder isoliert frei, mancbmal nur einzelne Glieder dieser Finger, ja eptl. nur auf der Volar- oder Dorsalseite. Es kommen vielerlei Variationen vor, die an zahlreichen Abbildungen demonstriert werden.

Während dieser Typus bisher nur an der Hand beschrieben wurde, hat G. ihn auch am Fuss beobachtet und zwar entweder am Fuss allein oder an der Hand und dem Fuss. In letzterem Falle betrifft die Störung dann immer die entsprechenden Partieu an Hand und Fuss, entweder die medialen oder die lateralen. Gewöhnlich sind dann auch die entsprechenden Partien an Unterarm. oder Unterschenkel befallen, so dass die Bilder denen beim näcbsten Typus ähno]n.

Zur Erklärung der Ausfälle dieses Typus ist anzunebmen, dass die mediale oder laterale Hälfte von Hand und Fuss in gesonderten Zentren 
vertreten sind im Sinne der erwähnten Empfindungsmechanismen. Die entsprechenden Abschnitte ron Hand und Fuss müssen ebenfalls kortikal zusammen lädierbaren Mechanismen entsprechen. Dass es sich dabei un einfach nebeneinander liegende Zentren handelt, ist unwabrscheinlich; wahrsebeinlieh handelt es sich um Mechanismen, deren Teile durch gemeinsame Funktion zu funktionellen Einheiten zusammengefasst sind, die deshalb bei einer funktionellen Schädigung als ganzes ausfallen gegenüber anderen erhaltenen Mechanismen. Die Gomeinsamkeit der Funktion liegt bei den gleichen Absohnitten von Hand und Fuss nicht innerhalb des menschlichen Daseins, sondern im Leben der tierischen Vorfahren in der Leistung des Vierfüsserganges. Dieser, beim Mensohen bedeutungslos gewordene Funktionszusammenhang ist kortikal so fest vertreten, dass er bei Störungen der Hirnrindenleistung in Erscheinung treten kann.

Neben diesen dureh gemeinsame Funktion bedingten Zusammenfassungen einzelner Abschnitte der Peripherie zu einheitlichen Gebilden müssen wir nach den Ausfällen noch relativ isolierte Zentren für kleinere Abschnitte annehmen, so z. B. für den Daumen und den kleinen Finger.

III. Typus: Der segmentale Typus. An dem Vorkommen der Störangen nach segmentalem T'ypus erscheint kein $Z$ weifel. Bemerkenswert ist 1., dass die Ausfälle nie einzelne Segmente oder einzelne Gruppen ron Segmenten isoliert, sondern dieselben nur - wenn auch sehr viel - stärker als die Umgebung betreffen, 2., dass fast immer die gleichen Segmente resp. die glaichen Gruppen von Segmenten befallen sind, und zwar an der oberen Körperhälfte C 8, D 1, D 2, D 3 ev. C 7, D 4; an der unteren L 5-S 3; ev. L 4 und S 2. Abweichungen kommen vor. Die Grenzen sind an einzelnen Stellen, z. B. an der Brust zwischen Brust- und Halssegmenten, am Gesäss zwischen Sakralund Brustsegmenten schare. Zur Erizärung dieser segmentalen Störungen ist einerseits daran zu denken, dass sie nur der Ausdruck der schon im Normalen bestehenden Empfindlichkeitsdifferenzen sind, die bei einer Herabsetzung der Leistungsfähigkeit der sensiblen Rindensphäre deutlicher in Erscheinung treten. Gewisse Erfahrungen sprechen dafür, dass dieses Moment mit eine Rolle spielt. Es ist aber auoh daran zu denken, dass gewisse Segmentgruppen eine gemeinsame Vertretung in der Hirnrinde haben und so gemeinsam lädiert werden können. Als Ursache hierfür ist ebenfalls wieder das kortikale Beziebungen and Zusammenfassungen schaffende Moment der gemeinsamen Funktion ins Auge zu fassen, obne dass bisher über diese funltionelle Gemeinsamkeit der betroffenen Segmentgruppen schon etwas bestimmtes zu sagen wäre.

Zwischen diesen Haupttypen der Störungen kommen verschiedene Kombinationen vor. Das so oft recht komplizierte Bild wird noch dadurch verwickelter, dass einzelne Abschnitte nur für einzelne Qualitäten besonders unterund überempfindlich sind, dass ferner in manchen Abschnitten die Verschiedenartigkeit des Reichtums der peripheren Innervation Differenzen schafft (so z. B. die doppelseitige Innervation in der Nähe der Mittellinie), die in den Sensibilitätsbildern ihren Ausdruck finden. Ausgedehnte Nachuntersuchungen sind 
erforderlich. Sie werden uns nicht nur über den speziellen Aufbau des sensiblen Rindenabschnitts Aufklärung bringen, sondern ein wertvolles Material für die Auffassung der Rindenorganisation ganz im allgemeinen liefern. Als wesentliches allgemeines Ergebnis seiner Untersuchung hebt Goldstein hervor die Tatsache, dass die tatsächlichen Befunde der Ausbreitung der Sensibilitätsstörungen nur verständlich werden unter weitgehender Berücksichtignng des funktionellen Momentes, das für den Aufbau der Rindenorganisation von fundamentaler Bedeutung ist. Er kommt so bei der Auffassung der sensiblen Störungen zu der gleichen Auffassung, die er bei der Betrachtung der aphasischapraktischen Störungen vertreten hat.

(Eigenbericht.)

24. Herr Gierlich-Wiesbaden: „Die Ausbildung der Grosshirnbahnen bei Mensch und Tier."

Vortragender berichtet summarisoh über Untersuchungen, die er und Breymann an der Schnittseriensammlung des Frankfurter Neurologischen Instituts angestellt haben, um zu ermitteln, nach welchen Gesichtspunkten die Ausbildung der Grosshirnbahnen in der Säugetierreihe erfolge. Vortragender hat diese Bahnen dort, wo sie als geschlossene Fussbahnen aus dem Gehirn austreten, gemessen, ihr Grössenverhältnis zum Gesamthirnschenkel festgestellt und so einen Ueberblick erhalten über die Fussbahn bei den einzelnen Tieren. Bei den untersuchten Insektenfressern war die Fussbahn auf Weigertschnitten blass und reichte manchmal nicht bis ins Rückenmark. Hier ist die phylogenetisch älteste Anlage der Bahn. Der biologische Grund ihrer Fntwioklung ist hente nicht erkennbar. Im übrigen war die Fussbahn bei primitiven Tieren am geringsten entwickelt, bei anderen Tieren derselben Ordnung dagegen oft auffallend gut. Die Ausbildung richtet sich somit nicht nach der Stellung der Tiere im System, sondern nur nach biologischen Bedürfnissen. Vortragender ermittelte ferner durch Ausmessung der Pyramiden liurz vor der Kreuzung als Rückenmarksanteil der Fussbahn ca. 30 bis 40 pCt., als Kleinhirnanteil 60 bis 70 pCt. Diese Grosshirnbahnen strahlen in die Kleinhirnhemisphären ein, während der Wurm ein phylogenetisch älteres Gebilde darstellt. Der ziemlich konstante, relativ hohe Prozentsatz zeigt die Wichtigkeit der Grosshirn-Kleinhirn-Bahnen. Kleist bringt ihren Ausfall mit Muskelsteifigkeit und Kontraktionsnachdauer der willkürlichen Bewegungen in Verbindung. In der untersuchten Tierreihe war der Anteil des Kleinhirns an der Fussbahn weit unter den Mittelwerten von 60 bis 70 pCt. bei Tieren mit fast kriechender ungelenker Fortbewegung, dagegen überschritt er die Mittelwerte bedeutend bei Tieren mit auffaliend flinken und gewandten Körperbewegungen in den verschiedenen Ordnangen, so z. B. bei Eichhörnchen, Hund, Pferd, dann bei Affen und dem Menschen. Vielleicht liegt hier ein Zusammenhang vor, der die Auffassung Kleist's stützen könnte. Breymann ermittelte an denselben Schnittserien den Pyramidenanteil am Rückenmark. Beim Menschen beträgt derselbe etwa 30 pCt., bei den untersuchten Affen 20 bis 13 pCt. Dieser hohe Prozentsatz ist bedingt durch die rom Grosshirn abhängigen Einzelbewegungen der Finger und Zehen. Es fo]gen dann aber zwei geistig niedrigstehende Tiere mit 
relativ hohen Prozentzahlen, Murmeltier und Stachelschwein. Hier liegen besondere biologische Gründe ror. Beim Stachelschwein findet der hohe Prozentsatz seine Erklärung in der biologisch wichtigen Innervation eines mächtigen Hautmuskels zur Bewegung des Stachelpanzers. Andere Nager weisen dementsprechend viel geringere Prozentzahlen auf. Bei den Robben, die Ballspiel erlernen können, ist die Prozentzabl recht hoch, bei den Raubtieren noch ca. 6 bis 8 pCt., boi dem Elephanten und den Huftieren mit ihren plumpen Füssen und minimalen Zehenbewegungen sehr gering. „Die Ausbildung der Grosshirnbahnen bei Mensch und Tier richtet sich nach den biologischen Anforderungen, die an dieselben gestellt werden. Eine Gegenüberstellung der anatomischen Befunde mit besonderen physiologischen and biologischen Fähigkeiten der Tiere dürfte wohl imstande sein, unsere Kenntnisse über die Leistungen der Grosshirnbahnen in dieser oder jener Richtung zu fördern."

(Die ausfübrlichen $A$ rbeiten im Anat. Anz., Bd.48/49.)

(Eigenbericht.)

25. Herr Wilb. Neumann, z. Z. Baden-Baden: „Ueber Pseudopsychologie der Tiere. Versuche mit dem "denkenden Hunde" in Mannhejm."

Der sogenannte denkende Hund Rolf wurde durch seine Umgebung veranlasst, sich zur angeblichen Aeusserung seiner Gedanken eines mechanischen Verfahrens zu bedienen, das von vornherein die Einmischung der Gedanken seiner Umgebung nicht ausschloss. Dass eine solche Einmischung tatsächlich stattgefunden hat, liess sich durch zahlreiche Versuche nachweisen. Wurde die Einmischung menschlicher Gedanken experimentell ausgeschlossen, so blieben der dem Hunde gestellten Aufgabe entsprechende Hundegedanken stets aus. Bei entsprechenden Vexierversuchen zeigte es sich, dass statt der ausgebliebenen Hundegedanken solche Menschengedanken einzutreten pflegten, von denen die Umgebung des Hundes irrtümlich glaubte, dass sie als Beweis für die gerade geforderten selbständigen Verstandesleistungen des Hundes tauglich wären. Alle bisher protokollierten „Denkleistungen" des Mannheimer Hundes müssen demnach bis zum Beweise des Gegenteils als Denkleistungen seiner Umgebung angesprochen werden, und es ist wahrsoheinlich, dass auch an der Registrierung dieser Gedanken dem Hunde kein seelischer Anteil zuzuschreiben ist, sondern nur ein rein mechanischer. Die auf Grund der "Mannheimer Tatsachen" in der Literatur veröffentlichten Erörterungen fallen somit porläufig aus dem Rahmen der Tierpsychologie. Ein Hund, dessen Denken sich nachweislich in sprachlichen Formen zu äussern vermag, bleibt ebenso zu finden, wie überhaupt ein Hund, dessen Seelenleben sich grundsätzlich über das den Psychologen, Liebhabern und Züchtern seit langem vertraute Gebiet hinaus erstreckt.

(Erscheint ausfïhrlich in der Münch. med.Wochenschr.) (Eigenbericht.)

26. Herr Steiner-Strassburg: "Das Zentralnervensystem bei der Hühnerspirochaetose, ein Beitrag zur vergleichenden Pathologie der Syphilis des Zentralnervensystems."

Die experimentelle Hühnerspirochaetose wird ausgiebig benützt bei der Erforsehung der Chemotherapie der Spirillosen. Vortr. schildert zunächst die 
Methode der Einimpfung, dann das klinische Bild der Erkrankung, die mit einer Krisis endigt. Bei der Krisis und kurz vorher verschwinden die Spirochaeten aus dem Blut. Bisher wurde allgemein ein Zugrundegehen der Spirochaeten in der Gefässbahn angenommen.

Vortr. konnte nun im Gehirn sowohl wie im Rückenmark der in der Krisis getöteten Hühner ein Austreten der Spirochaeten aus der Blutbahn, oft in Agglomerationen, nachweisen. Der Untergang der Spirochaeten findet nicht selten im adventitiellen Gefässraum statt. Häufiger aber finden sich einzelne Spirochaeten im freien Gewebe des zentralen Nervensystems, manchmal in ibrer gewöhnlichen Form etwas verändert, also wohl schon im Beginn des Absterbens.

Bei Tötung vor der Krisis sind die Spirochaeten nur in den Gefässbahnen des Zentralnervensystems zu sehen.

Auffällig und wichtig ist, dass die in der Krisis aus den Gefässen gewanderten Spirochaeten häufig sich in den Ganglienzellen nachweisen lassen, wo sie ebenfalls sich in ihrer Form verändern. In manchen Zollen lassen sich als Ueberbleibsel der Spirochaeten nur mehr fein verteilte, punktförmige, geschwärzte Pigmentansammlungen nachweisen, in anderen Zellen dagegen sind die Spirocbaeten, die gelegentlich in zwei Exemplaren beisammen liegen, deutlich erkennbar.

Die Spirochaeten liegen nur im Zellleib, ein Eindringen in den Kern ist nie beobachtet worden.

Kurz werden die Reaktionserscheinungen des nervösen Gewebes besprochen.

Die Bedeutung des Uebergangs der Spirochaeten aus dem Gefässlumen in das Parenchym ist gross.

Wir haben damit eine Erklärung für diə in der Krisis bei den meisten Tieren auftretenden Krämpfe und für die Somnolenz in der Krisis.

Wir verstehen ferner die bei manchen Tieren nach Ueberstehen der Krisis einsetzenden Lähmungen.

Endlich ist das Resultat der Untersuchungen wichtig für die Chemotherapie der Spirillosen: nicht dasjenige Mittel ist das ideale, das die Spirochaeten in der Blutbahn zum Verschwinden bringt, indem es sie aus der Blutbahn vertreibt; sondern von einem zweckmässigen Mittel ist zu verlangen, dass die Spirochaeten in der Blutbahn so abgetötet werden, dass ihnen die Möglichkeit aus der Blutbahn auszutreten, genommen ist.

Bei dem chemotherapeutischen Hühnerspirilloseversuch sind also immer anatomische Untersuchungen hinsichtlich des Austritts von Spirochaeten aus der Blutbahn mitheranzuziehen.

Bei der Syphilis müssen wir ein lymphotropes, ein haemotropes und ein parenchymotropes Stadium des Erregers untersoheiden. Gerade im baemotropen Stadium, in der sog. Dispersionsperiode, kurz vor und während des Auftretens des sekundären Exanthems ist eine geeignete und eingehende Therapie sehr am Platz.

Vor allem aber ist die Anwendung von Mitteln, die einen Uebergang aus der.Blutbahn in das umgebende Gewebe erleichtern können, peinlichst zu vermeiden. 
286 41. Wandervers. der Südwestdevtschen Neurologen u. Irrenärzte.

Die Prüfung, wie sich entzündungserregende Mittel hinsichtlich ibrer Einwirkung auf die in der Blutbahn kreisende Spirochaete gallinarum verhalten, ob sie einen Austritt aus der Gefässbahn erleichtern oder erschweren, wäre auch ausserordentlich wichtig, gerade auch im Hinblick auf die Paralysetherapie mit Natrium nucleinicum und Tuberkulin.

Die Versuche sind vor Kriegsbeginn unternommen worden und konnten nicht mebr weitergeführt werden.

Der Vortrag wird ausführlich anderweitig veröffentlicht.

(Eigenbericht.) reaktion."

27. Herr Weichbrodt-Frankfurt/Main: "Eine einfache Liquor-

Der Vortragende berichtet über eine einfache Liquorreaktion, die darin besteht, dass man von einer Sublimatlösung $1 / 10003$ T'eile zu 7 Teilen Liquor zusetzt; wäbrend der normale Liquor klar bleibt, zeigt der pathologische Liquor eine deutliche Trübung, die meist sofort auftritt, bei schwacher Reaktion spätestens nach 2-3 Minuten. Der Vortragende fand die Reaktion nur bei den durch Lues bedingten Hirnerkrankungen positiv. Meningitis und andere organische nichtluetische Erkrankungen des Nervensystems standen ihm nur in geringer Zahl zur Verfügung, da die Versuche nur an dem Material der Irrenklinik gemacht wurden.

Auch bei Goldchlorid fand der Vortragende ähnliohe Verhältnisse. Eine Goldchloridlösung $1 / 100$ gibt in derselben Versucbsanordnung ein ähnliches Resultat, und nicht nur in einer Lösung $1 / 100$, sondern anch $1 / 1000$, nur mit dem Unterschied, dass bei der Goldchloridlösung 1/1000 der Liquor Farbveränderungen eingeht, die typisch für die positive und negative Reaktion sind. Die Resultate decken sich mit der Goldsolreaktion von Lange, so dass die vom Vortragenden angeführte Reaktion ibrer Einfachheit wegen vielleicht vorzuziehen ist.

(Eigenbericht).

28. Herr Jahnel-Frankfurt/Main: "Ueber einige interessante Spiroebaetenbefunde bei Paralyse."

Vortragender demonstrierte Lichtbilder des Treponema pallidum von gefärbten Ausstrichen des Hirnbreis von Paralytikern (Lumière-Photographien), die der photographische Laborant der Frankfurter Klinik, Herr Rudolph, angefertigt batte. Ferner besprach der Vortragende an Photographien einige interessante morphologische Erscheinungen beim Treponema pallidum in der Pia, in der obersten Rindenschicht, sowie in grossen Massen an den Gefässen, an welchen Stellen Noguchi nie Treponemen geseben hatte. In zahlreichen Ganglienzellen fanden sich Treponemen der verschiedensten Form, ebenso in anderen Formelementen des Nervensystems (Stäbchenzellen).

Vortragender wies anf die Bedeutung umfassender Spirochaetenuntersuchungen bei Paralyse hin und erwähnte einen klinisch nicht erkannten Fall von Paralyse, in welchem er bei der Sektion durch den Treponemanachweis im Dunkelfelde die Diagnose stellen konnte.

(Eigenbericht.) 
29. Herr Mörchen-Wiesbaden: "Ueber traumatische Neurosen bei Kriegsgefangenen."

Die hier vor einem Jahr yon Liliens tein gemachte vorläufige Mitteilung über das fast vollständige Fehlen ,traumatischer Neurosen" bei Kriegsgefangenen kann jetzt auf Grund $11 / 2$ jähriger systematischer Feststellungen (vor allem an den neverdings von Verdun her das Darmstädter Durchgangslager passierenden Gefangenen) von dem Vortragenden durchaus bestätigt werden. Im ganzen kommt ein Zahlenmaterial von über 60000 Gefangenen in Betracht. Davon hat. der Vortragende ca. 40000 genügend eingehend ärztlich besichtigt, um feststellen za können, dass auch bei den zahlreichen aus schwerstem Trommelfeuer kommenden französischen Gefangenen von Verdun hor trotz Verschïtung, Minenexplosion usw. im Lager die Bilder der traunatischen Neurosen fast rollständig fehlten. Dabei wurde durch gruppenweises Befragen der Gefangenen in Erfahrung gebracht, dass die Folgen somatischer Erschütterung und psychischer Shocks in verschiedenartigen individuellen psychoneurotischen Reak-. tionen vor der Gefangennahme bei ihnen wobl zum Ausdruck gekommen waren. Aber es kam nicht mehr zu einer Fixierung der Erscheinungen. Augenscheinlich. schafft die Gefangenschaft eine psychische Situation, die der Konservierung resp. nachträglichen Entwicklung psychomotorischer und ysychosensibler Shockwirkungen ungünstig ist. Die psychische (quasi hypnotische) Einstellung. auf bestimmte Innervationsblockierungen wird nicht gefördert resp. sie wird gestört. Ueber die einzelnen hier wirksam werdenden psychischen Bedingtheiten. wird in einer demnächstigen Veröffentlichung des Vortragenden Näheres mitgeteilt werden. Es handelt sich nicht $\mathrm{n}$ ur um das Fehlen gewisser Begehrungsoder verdrängter Angstvorstellungen. So viel ist aber schon jetzt zu sagen: Bei unseren Bildern der "traumatischen Neurosen" in ihrer Vielgestaltigkeit ist jedenfalls das, was wir bei Gefangenen gar nicht zu sehen bekommen, nicht organisch bedingt.

(Eigenbericht.)

Freiburg i. B. und Strassburg, Angust 1916.

Hauptmann. Steiner. 